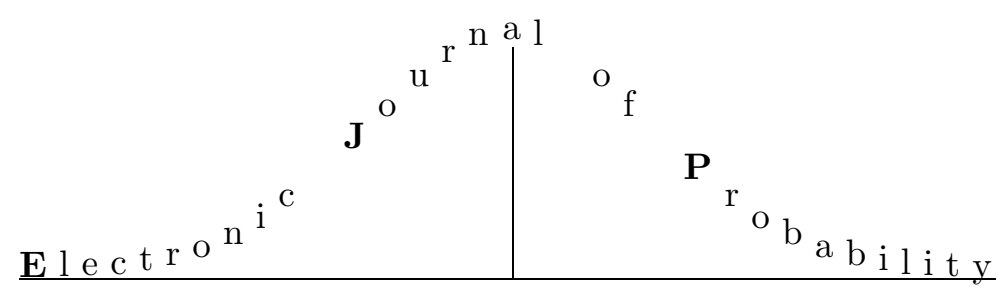

Vol. 9 (2004), Paper no. 23, pages 674-709.

Journal URL

http://www.math.washington.edu/ ejpecp/

\title{
A General Analytical Result for Non-linear SPDE's and Applications
}

\section{Laurent Denis}

Université du Maine, Faculté des Sciences,

Département de Mathématiques,

Laboratoire de Statistique et Processus,

Avenue Olivier Messiaen,

72085 Le Mans CEDEX 9, France

e-mail: ldenis@univ-lemans.fr

and

\section{Stoica}

University of Bucharest, Faculty of Mathematics,

Str. Academiei 14, Bucharest, RO -70109, Romania

e-mail: lstoica@pompeiu.imar.ro

\begin{abstract}
Using analytical methods, we prove existence uniqueness and estimates for s.p.d.e. of the type

$$
d u_{t}+A u_{t} d t+f\left(t, u_{t}\right) d t+R g\left(t, u_{t}\right) d t=h\left(t, x, u_{t}\right) d B_{t},
$$

where $A$ is a linear non-negative self-adjoint (unbounded) operator, $f$ is a nonlinear function which depends on $u$ and its derivatives controlled by $\sqrt{A} u, R g$ corresponds to a nonlinearity involving $u$ and its derivatives of the same order as $A u$ but of smaller magnitude, and the right term contains a noise involving a $d$-dimensional Brownian motion multiplied by a nonlinear function. We give a neat condition concerning the magnitude of these nonlinear perturbations. We also mention a few examples and, in the case of a diffusion generator, we give a double stochastic interpretation.
\end{abstract}


MSC: 60H15; 60G46; 35R60

Keywords: Stochastic Partial Differential Equations, Dirichlet space, Bilaplacian, Doubly stochastic representation.

Submitted to EJP on December 18, 2003. Final version accepted on August 30, 2004. 


\section{Introduction}

The starting point of this work is the following stochastic partial differential equation in divergence form:

$$
\begin{gathered}
d u_{t}-\sum_{i, j=1}^{N} \partial_{i}\left(a^{i, j} \partial_{j} u_{t}\right) d t+\widetilde{f}\left(t, x, u_{t}, \frac{1}{\sqrt{2}} \nabla u_{t} \sigma\right) d t \\
+\sum_{i=1}^{N} \partial_{i}\left(a^{i j} \widetilde{g}_{j}\left(t, x, u_{t}, \frac{1}{\sqrt{2}} \nabla u_{t} \sigma\right)\right) d t=\sum_{i=1}^{d} \widetilde{h}_{i}\left(t, u_{t}, \frac{1}{\sqrt{2}} \nabla u_{t} \sigma\right) d B_{t}^{i},
\end{gathered}
$$

where $a=\frac{1}{2} \sigma \sigma^{*}$ is a symmetric non-negative definite matrix valued function defined from $\mathbf{R}^{N}$ into $\mathbf{R}^{N \times N}, B=\left(B^{1}, \ldots, B^{d}\right)$ is a $d$-dimensional Brownian motion and $\widetilde{f}, \widetilde{g}, \widetilde{h}$ are only assumed to be Lipschitz continuous. We prove existence, uniqueness and estimate the solution (with initial condition) under very weak conditions on the entries of $a$. They may be discontinuous and even degenerated.

Due to the analytical methods we use, we see that in fact one can solve a s.p.d.e. in a much more general context, namely

$$
d u_{t}+A u_{t} d t+f\left(t, u_{t}\right) d t+R g\left(t, u_{t}\right) d t=\sum_{i=1}^{d} h_{i}\left(t, u_{t}\right) d B_{t}^{i}
$$

where $A$ is a self-adjoint non-negative operator defined on a Hilbert space, $f, g, h$ are adapted random functions defined on $[0,+\infty[\times F$ where $F$ is the domain of $A^{1 / 2}$ and $R g$ corresponds to a term of the same degree as $A$, but of lower magnitude in some sense. The functional approach makes things clearer and it also has the advantage of including as particular cases other examples, like for instance the bi-laplacian in $\mathbf{R}^{N}$ or the Ornstein-Uhlenbeck operator in infinite dimension.

The plan of the paper is as follows. In Section 2 we set notations, hypotheses and we announce the main results. In Section 3 we treat the relevant deterministic equations and in Section 4 we prove existence, uniqueness and estimates in terms of the data of the solution of the equation (2). Section 5 is devoted to some examples. Finally, in the last section we give the probabilistic interpretation to the solutions of the above equation (1) under the supplementary condition of uniform ellipticity.

\section{Notations, hypotheses and main results}

\subsection{Hilbert framework and hypotheses}

Let $(H,(\cdot, \cdot))$ be a separable Hilbert space whose norm is simply denoted by $\|\cdot\|$ and $\left(P_{t}\right)$ a symmetric strongly continuous semigroup on $H$. Let 
$(A, \mathcal{D}(A))$ be the infinitesimal generator of the semigroup. It is a nonnegative, densely defined, self-adjoint operator. The operator $A^{1 / 2}$ will play a special role in what follows, so that we introduce the notation $F=$ $\mathcal{D}\left(A^{1 / 2}\right), \mathcal{E}(u, v)=\left(A^{1 / 2} u, A^{1 / 2} v\right)$ and $\mathcal{E}(u)=\left\|A^{1 / 2} u\right\|^{2}$ for $u, v \in F$. The usual norm on $F$ is given by $\|u\|_{F}^{2}=\mathcal{E}(u)+\|u\|^{2}$, and we recall that $F$ is a Hilbert space with respect to this norm. The space $\mathcal{D}(A)$ is also considered as a Hilbert space endowed with the norm defined by $\|u\|_{\mathcal{D}(A)}^{2}=\|A u\|^{2}+\|u\|^{2}$.

The aim of this paper is to study the solutions of the nonlinear stochastic partial differential equation of parabolic type $(2)$, where $\left(B_{t}\right)$ represents an adapted $d$-dimensional Brownian motion defined on a standard filtered probability space $\left(\Omega, \mathcal{F}, \mathcal{F}_{t}, P\right)$. The letter $R$ denotes a bounded linear operator $R: K \rightarrow F^{\prime}$ defined on some Banach space $K$, with values in the dual $F^{\prime}$ of $F$, and it is assumed to map a dense subspace $J$ of $K$ into $H$. We shall adopt the usual convention that $F \hookrightarrow H \hookrightarrow F^{\prime}$, so that we may write this condition in the form $R J \subset H$. The letters $f, g, h$ denote time dependent random functions which depend on the unknown solution $u$ in a nonlinear manner. They are defined as follows:

$$
\begin{array}{lll}
f: & \mathbf{R}_{+} \times \Omega \times F \rightarrow H, \\
g & : & \mathbf{R}_{+} \times \Omega \times F \rightarrow K, \\
h: & \quad \mathbf{R}_{+} \times \Omega \times F \rightarrow H^{d},
\end{array}
$$

where $H^{d}=H \times \cdots \times H$ denotes the cartesian product of $d$ copies of $H$. These functions are assumed to be predictable and to satisfy the following Lipschitz conditions

$$
\begin{aligned}
\|f(t, \omega, u)-f(t, \omega, v)\| & \leq C\|u-v\|_{F} \\
\|g(t, \omega, u)-g(t, \omega, v)\|_{K} & \leq C\|u-v\|+\alpha \mathcal{E}(u-v)^{1 / 2} \\
\|h(t, \omega, u)-h(t, \omega, v)\|_{d} & \leq C\|u-v\|+\beta \mathcal{E}(u-v)^{1 / 2}
\end{aligned}
$$

for $u, v \in F, t \geq 0$ and almost all $\omega \in \Omega$, where $C, \alpha, \beta$ are positive constants. The space $H^{d}$ is equipped with the product norm:

$$
\forall x=\left(x_{1}, \ldots, x_{d}\right) \in H^{d},\|x\|_{d}=\sqrt{\left\|x_{1}\right\|^{2}+\ldots+\left\|x_{d}\right\|^{2}} .
$$

The constants $\alpha$ and $\beta$ should satisfy the following condition:

$$
2 \alpha\|R\|+\beta^{2}<2,
$$

where $\|R\|$ denotes the norm of the linear operator $R$. This means that the size of the second degree perturbation introduced by $R$ and the first degree perturbation associated with the Brownian motion, should be relatively small. Indeed, in the case of the equation (1), the term $R g(u)$ contains second order derivatives of the unknown function $u$ (see Subsection 5.1). But, 
if the constant $\alpha$ is small, then the linear term $A u$ controls the nonlinear term $R g(u)$. E.g. if one assumes that $h \equiv 0$, then the equation (1) becomes a deterministic parabolic equation and the condition (H1), with $\beta=0$, becomes the usual condition which ensures that the problem is well posed. On the other hand, the fact that the constant $\beta$ should be small is well-known in the context of doubly stochastic BSDE (see [15]). In fact, E. Pardoux proved in [14] that a condition on $\beta$ is necessary for (1) to be a well-posed stochastic parabolic equation.

Moreover it is assumed that

$$
\begin{aligned}
& f(\cdot, \cdot, 0) \in L_{l o c}^{2}\left(\mathbf{R}_{+} \times \Omega ; H\right), \\
& g(\cdot, \cdot, 0) \in L_{l o c}^{2}\left(\mathbf{R}_{+} \times \Omega ; K\right), \\
& h(\cdot, \cdot, 0) \in L_{l o c}^{2}\left(\mathbf{R}_{+} \times \Omega ; H^{d}\right),
\end{aligned}
$$

where $L_{l o c}^{2}\left(\mathbf{R}_{+} \times \Omega ; H\right)$ denotes the set of $H$-valued functions $u$ such that for all $T \geq 0, u \in L^{2}([0, T] \times \Omega ; H)$.

Throughout this paper, we will assume that the hypotheses (H1), (H2), (H3) are satisfied.

\subsection{Notations}

If $(a, b)$ is an open interval we will use the notation $L_{l o c}^{2}((a, b) ; H)$ to denote the space of all functions $u:(a, b) \rightarrow H$ such that

$$
\int_{r}^{s}\left\|u_{t}\right\|^{2} d t<\infty
$$

for any closed interval $[r, s] \subset(a, b)$. The space $L_{l o c}^{2}\left(\mathbf{R}_{+} ; H\right)$ will consist of all measurable maps $u: \mathbf{R}_{+} \rightarrow H$ such that all the following quantities are finite

$$
\int_{0}^{T}\left\|u_{t}\right\|^{2} d t<\infty, T>0
$$

Similar spaces will be considered with $H$ replaced by $F$ and $\mathcal{D}(A)$. We denote by $\widetilde{F}$ the subspace of $L_{l o c}^{2}\left(\mathbf{R}_{+} ; F\right)$ consisting of all maps $u$ that are $H$-continuous and endow it with the seminorms

$$
\|u\|_{T}:=\left(\sup _{0 \leq t \leq T}\left\|u_{t}\right\|^{2}+2 \int_{0}^{T} \mathcal{E}\left(u_{t}\right) d t\right)^{1 / 2}, T>0 .
$$

The space of test functions in our study will be $\mathcal{D}=\mathcal{C}_{c}^{\infty}\left(R_{+}\right) \otimes \mathcal{D}(A)$, the algebraic tensor product of the space of infinite differentiable functions on $[0, \infty)$ vanishing outside a finite interval with the domain of the infinitesimal operator $A$. Since $\mathcal{C}_{c}^{\infty}\left(R_{+}\right)$is dense in $L_{l o c}^{2}\left(\mathbf{R}_{+}\right)$and $\mathcal{D}(A)$ is dense in $F$ and in $H$, it follows that $\mathcal{D}$ is dense both in $L_{l o c}^{2}\left(\mathbf{R}_{+} ; F\right)$ and in $L_{l o c}^{2}\left(\mathbf{R}_{+} ; H\right)$. 
We now introduce some spaces of processes defined on our given probability space. We denote by $\mathcal{P}(H)$ the space of all predictable processes $u: \mathbf{R}_{+} \times \Omega \rightarrow H$ for which the quantities

$$
E \int_{0}^{T}\left\|u_{t}\right\|^{2} d t, T>0
$$

are all finite. Factorizing the space $\mathcal{P}(H)$ by its subspace consisting of processes for which the above quantities vanish we get a subspace of $L_{l o c}^{2}\left(\mathbf{R}_{+} \times \Omega ; H\right)$. Similarly, $\mathcal{P}(F)$ denotes the space of all predictable processes $u: \mathbf{R}_{+} \times \Omega \rightarrow$ $F$ for which the quantities

$$
E \int_{0}^{T}\left(\left\|u_{t}\right\|^{2}+2 \mathcal{E}\left(u_{t}\right)\right) d t, T>0
$$

are finite. By factorization with respect to the subspace of nulls of the above quantities, $\mathcal{P}(F)$ becomes a subspace of $L_{l o c}^{2}\left(\mathbf{R}_{+} \times \Omega ; F\right)$. Finally we denote by $\widetilde{\mathcal{P}}(F)$ the sub-vector space of processes $u \in \mathcal{P}(F)$ with $H$-continuous trajectories, that is, $u(\cdot, \omega) \in \widetilde{F}$ almost surely. This space will be endowed with the seminorms

$$
\left(E\|u\|_{T}^{2}\right)^{1 / 2}, T>0 .
$$

The space $\widetilde{\mathcal{P}}(F)$ is the basic space in which we are going to look for solutions.

The conditions (H1),(H3) imposed to $f, g, h$ imply that if $u \in \mathcal{P}(F)$, then $f(\cdot, u),. h_{i}(\cdot, u.) \in \mathcal{P}(H)$ for all $i \in\{1, \ldots, d\}$ and $g(\cdot, u$. $)$ is predictable and belongs to the space $L_{l o c}^{2}\left(\mathbf{R}_{+} \times \Omega ; K\right)$. In particular we may define the solution of the stochastic partial differential equation as follows.

Definition 1 We say that $u \in \mathcal{P}(F)$ is a weak solution of the equation (2) with initial condition $u_{0}=x \in L^{2}\left(\Omega, \mathcal{F}_{0}, P ; H\right)$ if the following relation holds almost surely, for each $\varphi \in \mathcal{D}$,

$$
\begin{gathered}
\int_{0}^{\infty}\left[\left(u_{s}, \partial_{s} \varphi\right)-\mathcal{E}\left(u_{s}, \varphi_{s}\right)-\left(f\left(s, u_{s}\right), \varphi_{s}\right)-\left(\operatorname{Rg}\left(s, u_{s}\right), \varphi_{s}\right)_{F^{\prime}, F}\right] d s+ \\
+\sum_{i=1}^{d} \int_{0}^{\infty}\left(h_{i}\left(s, u_{s}\right), \varphi_{s}\right) d B_{s}^{i}+\left(x, \varphi_{0}\right)=0 .
\end{gathered}
$$

In fact, we will work with the notion of mild solution, which will be well-defined in section 4 (see Proposition 6).

Definition 2 We call $u \in \mathcal{P}(F)$ a mild solution of the equation (2) with initial condition $u_{0}=x$, if the following equality is verified almost surely, for each $t \geq 0$,

$$
\begin{array}{r}
u_{t}=P_{t} x-\int_{0}^{t} P_{t-s} f\left(s, u_{s}\right) d s-\int_{0}^{t} P_{t-s} R g\left(s, u_{s}\right) d s \\
+\sum_{i=1}^{d} \int_{0}^{t} P_{t-s} h_{i}\left(s, u_{s}\right) d B_{s}^{i} .
\end{array}
$$




\subsection{Main results}

The main results of this work are the following.

Theorem 2.1 The notions of mild and weak solutions coincide, moreover there exists a unique solution to equation (2) with initial condition $u(0)=$ $x \in L^{2}\left(\Omega, \mathcal{F}_{0}, P ; H\right)$. It belongs to $\widetilde{\mathcal{P}}(F)$ and satisfies the following estimate

$$
\begin{array}{r}
E\left(\sup _{0 \leq t \leq T}\left\|u_{t}\right\|^{2}+\int_{0}^{T} \mathcal{E}\left(u_{t}\right) d t\right) \\
\leq c E\left(\|x\|^{2}+\int_{0}^{T}\left(\|h(t, 0)\|_{d}^{2}+\|f(t, 0)\|^{2}+\|g(t, 0)\|_{K}^{2}\right) d t\right) .
\end{array}
$$

See Theorems 8 and 9 of Section 4. All along the proof, we make a special effort to get optimal conditions on the constants of Lipschitz continuity of the nonlinear terms. Our best result is that condition (H2) suffices to ensure the validity of the above theorem. Results of this kind for diffusion equations and even more precise information have already been obtained by Krylov [11], Mikulevicius and Rozovskii [13], and Göngy and Rovira [10]. However their methods do not seem to cover such general situations as our paper does (measurable coefficients, without strict ellipticity condition). One also has to mention the early work of Chojnowska-Michalik [5], which also treats SPDE 's in the general framework of Hilbert spaces, as we do. Our paper uses more or less the same type of arguments (e.g. contraction principle and semigroup properties), but we deal with two spaces: $H$ and $F$. Therefore we produce more precise estimates so that to take into consideration the Lipschitz continuity of the nonlinear terms with respect to both the norms of $H$ and $F$ (as it is stated in (H1)). And another thing that our paper brings is the neat condition (H2).

In Corollary 12 we give a probabilistic interpretation of the solution in the case of the concrete equation (1), where the coefficients $\left(a^{i j}\right)$ are assumed to be measurable and uniformly elliptic. That is, we show that the solution admits a doubly stochastic representation, in the sense that $u\left(t, X_{T-t}\right)$ is expressed as a sum of stochastic integrals with respect to $B$ and $X$, where $X$ is the process generated by $A$. The doubly stochastic representation was first proved by E. Pardoux and S. Peng in [15]. They proved it directly under different conditions and then used it to investigate the spde itself. Our result follows from an approximation result stated in Lemma 10, which shows that the solution of the linear stochastic equation is pathwise approximated by the solutions of certain deterministic linear equations. In fact these deterministic equations are obtained from the stochastic equation by replacing the Brownian with its usual polygonal approximation. The lemma holds in the general Hilbert space framework and may have other applications. 


\section{Preliminaries on the deterministic linear equa- tion}

In this section we introduce some notations and gather some facts concerning the deterministic linear equation, facts that are proved by standard methods from the theory of semigroups of operators (see [8]). For example it is wellknow that if $x \in H$, then for all $t>0, P_{t} x \in \mathcal{D}(A)$, and the map $t \rightarrow P_{t} x$ is $H$-continuous on $[0,+\infty[$ and $H$-differentiable on $] 0,+\infty[$ and its derivative is $\partial_{t} P_{t} x=-A P_{t} x$. (The semigroup is even analytic, but we will not use this fact.) We also have that $\forall u \in \mathcal{D}(A), \forall v \in F, \mathcal{E}(u, v)=(A u, v)$, which is useful when working with the notion of weak solution. We use several notions of weak solutions taken from [12].

The next lemma allows one to extend the space of test functions $\varphi$ permitted in the weak relations related to the solutions of evolution equations and their time derivatives.

Lemma 1 If $w: \mathbf{R}_{+} \rightarrow F$ has compact support and is $F$-differentiable with $F$-continuous derivative $\partial_{t} w$, then there exists a sequence $\left(w^{n}\right) \subset \mathcal{D}$ such that

$$
\begin{aligned}
\limsup _{n} \sup _{t}\left\|w_{t}^{n}-w_{t}\right\|_{F} & =0, \\
\lim _{n} \sup _{t}\left\|\partial_{t} w^{n}-\partial_{t} w\right\|_{F} & =0 .
\end{aligned}
$$

A similar approximation holds for $H$ valued functions.

Proof. Assume that $T>0$ is such that $w_{t}=0$, for each $t>T$. As $\mathcal{C}_{c}^{\infty}\left(R_{+}\right)$is dense in $\mathcal{C}_{c}\left(R_{+}\right)$and $\mathcal{D}(A)$ is dense in $F$, it is clear that there exists a sequence $\left(x^{n}\right)$ in $\mathcal{D}$ which converges uniformly to $\partial w$ in $F$ on $[0, T]$. We put

$$
\forall t \geq 0, w_{t}^{n}=w(0)+\int_{0}^{t} x_{s}^{n} d s
$$

which clearly is an element in $\mathcal{D}$. One verifies that

$$
\forall t \in[0, T],\left\|w_{t}^{n}-w_{t}\right\|_{F} \leq \int_{0}^{t}\left\|x_{s}^{n}-\partial_{s} w_{s}\right\|_{F} d s \leq T \sup _{t \in[0, T]}\left\|x_{t}^{n}-\partial_{t} w_{t}\right\|_{F} .
$$

The proof is complete.

Definition 3 A function $u \in L_{l o c}^{2}((a, b) ; H)$ is said to admit a weak derivative on the open interval $(a, b)$ if there exists a function $\partial_{t} u \in L_{l o c}^{2}((a, b) ; H)$ (that we shall call the weak derivative of $u$ ) which satisfies the relation

$$
\int_{a}^{b}\left(u_{t}, \partial_{t} \varphi\right) d t=-\int_{a}^{b}\left(\partial_{t} u_{t}, \varphi\right) d t
$$

for all test functions $\varphi \in \mathcal{D}$ such that $\varphi_{t}=0$ for $t$ outside a compact interval contained in $(a, b)$. 
Definition 4 Given $x \in H$ and $w \in L_{l o c}^{2}\left(\mathbf{R}_{+} ; H\right)$, an $\mathrm{s}$-weak solution of the linear equation

$$
\partial_{t} u+A u=w,
$$

with the initial condition $u(0)=x$, is a function $u \in L_{\text {loc }}^{2}((0, \infty) ; \mathcal{D}(A)) \cap$ $\mathcal{C}\left(R_{+}, H\right)$ which admits a weak derivative (in $\left.H\right)$ on $(0, \infty)$, such that the relation

$$
\partial_{t} u_{t}+A u_{t}=w_{t}
$$

is satisfied for almost all $t>0$, and such that $u_{0}=x$.

The $s$ in this name stands for the word "separate" and refers to the fact that in this definition the operator $\partial_{t}+A$ splits in the sum of two separate parts: $\partial_{t}$ and $A$. This is in contrast to another notion of weak solution we will introduce later on in the Definition 4 and to its stochastic version from the Definition 1.

The next lemma is the basic uniqueness result for the solutions considered in this paper. In particular it ensures uniqueness of $\mathrm{s}$-weak solutions of the above equation.

Lemma 2 If $u \in L_{l o c}^{2}\left(\mathbf{R}_{+} ; F\right)$ satisfies the relation

$$
\int_{0}^{\infty}\left[\left(u_{t}, \partial \varphi_{t}\right)-\mathcal{E}\left(u_{t}, \varphi_{t}\right)\right] d t=0
$$

with any $\varphi \in \mathcal{D}$, then $u \equiv 0$, as an element of $L_{l o c}^{2}\left(\mathbf{R}_{+} ; F\right)$.

Proof. Take $w \in \mathcal{D}$ and set $v_{t}=\int_{t}^{\infty} P_{s-t} w_{s} d s$. A direct verification shows that

$$
\partial_{t} v=A v_{t}-w_{t}
$$

Assume that $T$ is such that $w_{t}=0$ for $t \geq T$, and hence also $v_{t}=0$, for $t \geq T$. Then one has

$$
\begin{aligned}
-\int_{0}^{\infty}\left(u_{t}, w_{t}\right) d t & =\int_{0}^{\infty}\left(u_{t}, \partial v_{t}-A v_{t}\right) d t= \\
& =\int_{0}^{\infty}\left[\left(u_{t}, \partial v_{t}\right)-\mathcal{E}\left(u_{t}, v_{t}\right)\right] d t
\end{aligned}
$$

By the preceding lemma one gets a sequence $\left(v^{n}\right) \subset \mathcal{D}$ such that $v_{n} \rightarrow v$, uniformly in $F$ together with the derivatives. The last expression written with $v_{n}$,

$$
\int_{0}^{\infty}\left[\left(u_{t}, \partial v_{t}^{n}\right)-\mathcal{E}\left(u_{t}, v_{t}^{n}\right)\right] d t=0,
$$

vanishes according to the hypothesis. In the limit we have

$$
\int_{0}^{\infty}\left(u_{t}, w_{t}\right) d t=0
$$


Since $w$ is arbitrary, it follows that $u \equiv 0$.

The next proposition gives a formula for the solution of equation (3) and the basic estimates in terms of the data.

Lemma 3 If $x \in H$ and $w \in L_{l o c}^{2}\left(\mathbf{R}_{+} ; H\right)$ are given, then

$$
u_{t}=\int_{0}^{t} P_{t-s} w_{s} d s+P_{t} x, t \in \mathbf{R}_{+},
$$

is an $s$-weak solution of the linear equation (3), with initial condition $u_{0}=x$. Moreover $u \in \widetilde{F}$ and it satisfies the following relations, with any $T>0$,

$$
\begin{gathered}
\frac{1}{2}\left\|u_{T}\right\|^{2}+\int_{0}^{T} \mathcal{E}\left(u_{t}\right) d t=\int_{0}^{T}\left(w_{t}, u_{t}\right) d t+\frac{1}{2}\|x\|^{2}, \\
\|u\|_{T}^{2} \leq e^{T}\left(\|x\|^{2}+\int_{0}^{T}\left\|w_{t}\right\|^{2} d t\right) .
\end{gathered}
$$

Besides these, if $x \in F$, the solution satisfies the relation

$$
\mathcal{E}\left(u_{T}\right)+\int_{0}^{T}\left(\left\|\partial_{t} u\right\|^{2}+\left\|A u_{t}\right\|^{2}\right) d t=\mathcal{E}(x)+\int_{0}^{T}\left\|w_{t}\right\|^{2} d t .
$$

Proof. Fix $T>0$ and assume first that $w \in \mathcal{D}$ and $x \in \mathcal{D}(A)$. Then, it is clear that the maps $t \rightarrow P_{t} x, t \rightarrow \int_{0}^{t} P_{t-s} w_{s} d s$ belong to $C\left([0, T], \mathcal{D}\left(A^{3 / 2}\right)\right)$ and are $H$-differentiable with continuous derivatives. From this, we deduce that $t \rightarrow u_{t}$ belongs to $C([0, T], \mathcal{D}(A))$, it is also $H$-differentiable with continuous derivative and we have

$$
\forall t>0, \frac{d u_{t}}{d t}=w_{t}-A u_{t} .
$$

In particular it is an s -weak solution of (3) and moreover one can verify that $\partial_{t} u_{t}=\frac{d u_{t}}{d t}$ belongs to $F$.

Next we are going to establish the relations from the statement in this particular case. Integrating by parts, we have

$$
\int_{0}^{T}\left(\partial_{t} u_{t}, u_{t}\right) d t=\left\|u_{T}\right\|^{2}-\|x\|^{2}-\int_{0}^{T}\left(u_{t}, \partial_{t} u_{t}\right) d t
$$

so

$$
\begin{aligned}
\left\|u_{T}\right\|^{2}-\|x\|^{2} & =2 \int_{0}^{T}\left(\partial_{t} u_{t}, u_{t}\right) d t \\
& =2 \int_{0}^{T}\left(w_{t}-A u_{t}, u_{t}\right) d t \\
& =2\left(\int_{0}^{T}\left(w_{t}, u_{t}\right) d t-\int_{0}^{T} \mathcal{E}\left(u_{t}\right) d t\right)
\end{aligned}
$$


which is relation $(i)$. The relation $(i i)$ follows from this one by using the Schwartz inequality

$$
2 \int_{0}^{T}\left(w_{t}, u_{t}\right) d t \leq \int_{0}^{T}\left[\left\|w_{t}\right\|^{2}+\left\|u_{t}\right\|^{2}\right] d t
$$

and then applying Gronwall's lemma.

In order to deduce the third relation one starts with the following equality

$$
\left\|w_{t}\right\|^{2}=\left\|\partial_{t} u\right\|^{2}+\left\|A u_{t}\right\|^{2}+2\left(\partial_{t} u, A u_{t}\right),
$$

which is simply a consequence of (4). Then one integrates both sides of this equality. Since $2\left(\partial_{t} u, A u_{t}\right)=2 \mathcal{E}\left(\partial_{t} u, u_{t}\right)=\partial_{t} \mathcal{E}\left(u_{t}\right)$, one has

$$
\int_{0}^{T} 2\left(\partial_{t} u, A u_{t}\right) d t=\mathcal{E}\left(u_{T}\right)-\mathcal{E}(x),
$$

and so we get the relation $(i i i)$.

In order to obtain the result in the general case one approximates $x$ and $w$ with objects of the preceding kind. The three relations of the statement pass to the limit. The function $u_{t}^{\prime}=\int_{0}^{t} P_{t-s} w_{s} d s$ always belongs to $L_{l o c}^{2}\left(\mathbf{R}_{+} ; \mathcal{D}(A)\right)$. The function $u_{t}^{\prime \prime}=P_{t} x$ only belongs to $L_{l o c}^{2}((0, \infty) ; \mathcal{D}(A))$ in general. But this suffices to fulfil the requirements of the definition of an $\mathrm{s}$-weak solution.

Remark 1 The $s$-weak solution of the equation (3) always satisfies the relation

$$
u_{t}-u_{s}=-\int_{s}^{t} A u_{r} d r+\int_{s}^{t} w_{r} d r
$$

for any $0<s \leq t$. If $u(0)=x$ belongs to $F$, then the equality (iii) of the preceding proposition ensures that $A u_{r}$ is integrable up to 0. Therefore, in this particular case, the relation also holds with $s=0$ and one has, for each $t>0$,

$$
u_{t}=x-\int_{0}^{t} A u_{r} d r+\int_{0}^{t} w_{r} d r
$$

Now we are going to treat equations involving the operator $R$.

Definition 5 Let $x \in H, w^{\prime} \in L_{l o c}^{2}\left(\mathbf{R}_{+} ; H\right)$ and $w^{\prime \prime} \in L_{l o c}^{2}\left(\mathbf{R}_{+} ; K\right)$ be given. A weak solution of the equation

$$
\left(\partial_{t}+A\right) u=w^{\prime}+R w^{\prime \prime}
$$

with initial condition $u(0)=x$ is a function $u \in L_{l o c}^{2}\left(\mathbf{R}_{+} ; F\right)$ such that the next relation is fulfilled with any $\varphi \in \mathcal{D}$,

$$
\left(x, \varphi_{0}\right)+\int\left[\left(u_{t}, \partial_{t} \varphi\right)-\mathcal{E}\left(u_{t}, \varphi_{t}\right)+\left(w_{t}^{\prime}, \varphi_{t}\right)+\left(R w_{t}^{\prime \prime}, \varphi_{t}\right)_{F^{\prime}, F}\right] d t=0 .
$$


Clearly an s-weak solution of the equation (3) is also a weak solution of (5) with $w^{\prime}=w$ and $w^{\prime \prime}=0$. The uniqueness of the solution associated to given data follows again by Lemma 2 . The existence will also be obtained by an explicit formula. To treat the term containing $R$ we need to give a sense to the formal integral $\int_{0}^{t} P_{t-s} R w_{s} d s$ with $w \in L_{l o c}^{2}\left(\mathbf{R}_{+} ; K\right)$. This integral is well defined if $w \in \mathcal{C}_{c}^{\infty}\left(\mathbf{R}_{+}\right) \otimes J$, where $J$ is the space associated to $R$ in the introduction. Denote by $U: \mathcal{C}_{c}^{\infty}\left(\mathbf{R}_{+}\right) \otimes J \rightarrow \widetilde{F}$ the operator defined by

$$
(U w)_{t}=\int_{0}^{t} P_{t-s} R w_{s} d s, t \geq 0
$$

The next lemma proves that we can extend it by continuity. Later we will use the formal expression $\int_{0}^{t} P_{t-s} R w_{s} d s$ rather than $U w$.

Lemma 4 The operator $U$ admits a uniquely determined continuous extension

$$
U: L_{l o c}^{2}\left(\mathbf{R}_{+} ; K\right) \rightarrow \widetilde{F} .
$$

If $w \in L_{l o c}^{2}\left(\mathbf{R}_{+} ; K\right)$, then $u=U w$ is a weak solution of the equation

$$
\left(\partial_{t}+A\right) U=w, u(0)=0 .
$$

Moreover it satisfies the following relations,

$$
\begin{gathered}
\frac{1}{2}\left\|u_{T}\right\|^{2}+\int_{0}^{T} \mathcal{E}\left(u_{t}\right) d t=\int_{0}^{T}\left(R w_{t}, u_{t}\right)_{F^{\prime}, F} d t, \\
\|u\|_{T}^{2} \leq 2 e^{T}\|R\|^{2}\left(\int_{0}^{T}\left\|w_{t}\right\|_{K}^{2} d t\right) .
\end{gathered}
$$

Proof. One easily sees that if $w \in \mathcal{C}_{c}^{\infty}\left(\mathbf{R}_{t}\right) \otimes J$, then $R w \in \mathcal{C}^{\infty}\left(\mathbf{R}_{+}\right) \otimes H$. By Lemma 3, we deduce that $u=U w$ is an $\mathrm{s}$-weak solution of (3) with data $R w$ and initial condition $x=0$. ¿From relation $(i)$ of that lemma we get

$$
\frac{1}{2}\left\|U w_{T}\right\|^{2}+\int_{0}^{T} \mathcal{E}\left(U w_{t}\right) d t=\int_{0}^{T}\left(R w_{t}, U w_{t}\right) d t .
$$

Since, in general, one has $(x, y) \leq\|x\|_{F^{\prime}}\|y\|_{F}$, as soon as $x \in H$ and $y \in F$, the last integral is dominated by

$$
\|R\| \int_{0}^{T}\left\|w_{t}\right\|_{K}\left(\left\|U w_{t}\right\|^{2}+\mathcal{E}\left(U w_{t}\right)\right)^{1 / 2} d t
$$

Using Schwarz's inequality and then Gronwall's lemma one deduces the relation (ii) from the statement. The result with general $w \in L_{l o c}^{2}\left(\mathbf{R}_{+} ; K\right)$ follows by approximation.

Combining the preceding two lemmas one gets the following one. 
Lemma 5 The equation (5) admits a unique solution given by

$$
u_{t}=P_{t} x+\int_{0}^{t} P_{t-s} w_{s}^{\prime} d s+\int_{0}^{t} P_{t-s} R w_{s}^{\prime \prime} d s, t \in \mathbf{R}_{+} .
$$

It belongs to $\widetilde{F}$ and satisfies the following relations

$$
\begin{gathered}
\frac{1}{2}\left\|u_{T}\right\|^{2}+\int_{0}^{T} \mathcal{E}\left(u_{t}\right) d t=\int_{0}^{T}\left(\left(w_{t}^{\prime}, u_{t}\right)+\left(R w_{t}^{\prime \prime}, u_{t}\right)_{F^{\prime}, F}\right) d t+\frac{1}{2}\|x\|^{2}, \\
\|u\|_{T}^{2} \leq 2 e^{T}\left(\|x\|^{2}+\int_{0}^{T}\left\|w_{t}^{\prime}\right\|^{2} d t+\|R\|^{2} \int_{0}^{T}\left\|w_{t}^{\prime \prime}\right\|^{2} d t\right) .
\end{gathered}
$$

\section{Stochastic parabolic equations}

In what follows, we consider stochastic integrals of processes in $(\mathcal{P}(H))^{d}$ with respect to our Brownian motion. They have to be seen as Hilbert-space valued stochastic integrals. For this, we refer to Da Prato and Zabczyk [7], who deal with the more general case when the Brownian motion is also infinite dimensional. So, we know that this stochastic integral has good properties such as continuity in time, predictability and that a suitable version of Itô's formula holds.

One basic ingredient in the treatment of stochastic parabolic equations is the following result. Let us note that the assertion $(v)$ of this proposition was proved in [5] under more general conditions.

Proposition 6 Let $x \in L^{2}\left(\Omega, \mathcal{F}_{0}, P ; H\right), w=\left(w_{1}, \cdots, w_{d}\right) \in(\mathcal{P}(H))^{d}, w^{\prime} \in$ $\mathcal{P}(H)$ and $w^{\prime \prime} \in \mathcal{P}(K)$. We set

$$
\forall t \geq 0, u_{t}=P_{t} x+\sum_{i=1}^{d} \int_{0}^{t} P_{t-s} w_{i, s} d B_{s}^{i}+\int_{0}^{t} P_{t-s} w_{s}^{\prime} d s+\int_{0}^{t} P_{t-s} R w_{s}^{\prime \prime} d s .
$$

Then $u$ has a version in $\widetilde{\mathcal{P}}(F)$ and for each $\varphi \in \mathcal{D}$, it verifies the following weak sense relation almost surely,

$$
\begin{gathered}
\int_{0}^{\infty}\left[\left(u_{s}, \partial_{s} \varphi\right)-\mathcal{E}\left(u_{s}, \varphi_{s}\right)+\left(w_{s}^{\prime}, \varphi_{s}\right)+\left(R w_{s}^{\prime \prime}, \varphi_{s}\right)_{F^{\prime}, F}\right] d s \\
+\sum_{i=1}^{d} \int_{0}^{\infty}\left(w_{i, s}, \varphi_{s}\right) d B_{s}^{i}+\left(x, \varphi_{0}\right)=0 .
\end{gathered}
$$

Moreover, for all $t \geq 0$ :

$$
\left\|u_{t}\right\|^{2}+2 \int_{0}^{t} \mathcal{E}\left(u_{s}\right) d s=\|x\|^{2}+2 \sum_{i=1}^{d} \int_{0}^{t}\left(u_{s}, w_{i, s}\right) d B_{s}^{i}+
$$




$$
+\int_{0}^{t}\left(u_{s}, w_{s}^{\prime}\right) d s+\int_{0}^{t}\left(u_{s}, R w_{s}^{\prime \prime}\right)_{F, F^{\prime}} d s+\int_{0}^{t}\left\|w_{s}\right\|_{d}^{2} d s
$$

so

$$
\begin{aligned}
E\left\|u_{t}\right\|^{2} & +2 E\left(\int_{0}^{t} \mathcal{E}\left(u_{s}\right) d s\right)=E\|x\|^{2}+E\left(\int_{0}^{t}\left(u_{s}, w_{s}^{\prime}\right) d s\right)+ \\
& +E\left(\int_{0}^{t}\left(u_{s}, R w_{s}^{\prime \prime}\right)_{F, F^{\prime}} d s\right)+E\left(\int_{0}^{t}\left\|w_{s}\right\|_{d}^{2} d s\right) .
\end{aligned}
$$

And we have the estimate

$$
E\|u\|_{T}^{2} \leq c E\left(\|x\|^{2}+\int_{0}^{T}\left(\left\|w_{t}\right\|_{d}^{2}+\left\|w_{t}^{\prime}\right\|^{2}+\left\|w_{t}^{\prime \prime}\right\|_{K}^{2}\right) d t\right)
$$

where $c$ is a constant which only depends on $T$.

Finally, if $w^{\prime \prime}=0$, the random variables $\int_{0}^{t} u_{s} d s, t>0$, take value in $\mathcal{D}(A)$ and they satisfy the next relation almost surely

$$
u_{t}+A \int_{0}^{t} u_{s} d s=x+\sum_{i=1}^{d} \int_{0}^{t} w_{i, s} d B_{s}^{i}+\int_{0}^{t} w_{s}^{\prime} d s .
$$

Proof. We use the sequence of operators $P_{\frac{1}{n}}$ to construct a smoothing approximation

$$
w_{i, s}^{n}=P_{\frac{1}{n}} w_{i, s}, w_{s}^{\prime, n}=P_{\frac{1}{n}} w_{s}^{\prime}, z_{s}^{n}=P_{\frac{1}{n}} R w_{s}^{\prime \prime}, x^{n}=P_{\frac{1}{n}} x .
$$

Then $\left(w^{n}\right)$ is a sequence of elements in $\left(L^{2}([0, t] \times \Omega, \mathcal{D}(A))\right)^{d} \cap(\mathcal{P}(H))^{d}$ which converges to $w$ in $\left(L^{2}([0, t] \times \Omega, H)\right)^{d},\left(w^{\prime, n}\right)$ is a sequence of elements in $L^{2}([0, t] \times \Omega, \mathcal{D}(A))$ which converges to $w^{\prime}$ in $L^{2}([0, t] \times \Omega, H),\left(z^{n}\right)$ is a sequence in $L^{2}([0, t] \times \Omega, \mathcal{D}(A))$ which converges to $R w^{\prime \prime}$ in $L^{2}\left([0, t] \times \Omega, F^{\prime}\right)$, for all $t>0$, and $\left(x^{n}\right)$ converges to $x$ in $L^{2}(\Omega ; H)$. Define for all $n \in \mathbf{N}^{*}$,

$$
u_{t}^{n}=P_{t} x^{n}+\sum_{i=1}^{d} \int_{0}^{t} P_{t-s} w_{i, s}^{n} d B_{s}^{i}+\int_{0}^{t} P_{t-s} w_{s}^{\prime, n} d s+\int_{0}^{t} P_{t-s} z_{s}^{n} d s .
$$

We decompose $u$ and $u^{n}$ as

$$
\begin{gathered}
u_{t}=u_{t}^{1}+u_{t}^{2}+u_{t}^{3}, \\
u_{t}^{n}=u_{t}^{1, n}+u_{t}^{2, n}+u_{t}^{3, n},
\end{gathered}
$$

with

$$
u_{t}^{1}=\sum_{i=1}^{d} \int_{0}^{t} P_{t-s} w_{i, s} d B_{s}^{i}, u_{t}^{2}=P_{t} x+\int_{0}^{t} P_{t-s} w_{s}^{\prime} d s, u_{t}^{3}=\int_{0}^{t} P_{t-s} R w_{s}^{\prime \prime} d s,
$$


and

$u_{t}^{1, n}=\sum_{i=1}^{d} \int_{0}^{t} P_{t-s} w_{i, s}^{n} d B_{s}^{i}, u_{t}^{2, n}=P_{t} x^{n}+\int_{0}^{t} P_{t-s} w_{s}^{\prime, n} d s, u_{t}^{3, n}=\int_{0}^{t} P_{t-s} z_{s}^{n} d s$.

We first study the stochastic part. One easily sees that $u^{1, n}=P_{\frac{1}{n}} u^{1}$ and hence, it is not difficult to see that $u_{t}^{1, n}$ and $\int_{0}^{t} u_{s}^{1, n} d s$ belong to $\mathcal{D}(A)$, for all $t \geq 0$. Then we may perform the following calculations

$$
\begin{gathered}
A \int_{0}^{t} u_{s}^{1, n} d s=\int_{0}^{t} A u_{s}^{1, n} d s=\int_{0}^{t} \sum_{i=1}^{d} \int_{0}^{s} A P_{s-r} w_{i, r}^{1, n} d B_{r}^{i} d s=\sum_{i=1}^{d} \int_{0}^{t} \int_{r}^{t} A P_{s-r} w_{i, r}^{1, n} d s d B_{r}^{i}= \\
=-\sum_{i=1}^{d} \int_{0}^{t}\left(P_{t-r} w_{i, r}^{1, n}-w_{i, r}^{1, n}\right) d B_{r}^{i}=-u_{t}^{1, n}+\sum_{i=1}^{d} \int_{0}^{t} w_{i, r}^{1, n} d B_{r}^{i},
\end{gathered}
$$

which proves that $u^{n}$ has a version which is a $H$-valued semimartingale; as a consequence, it is $H$-continuous. Then, by Itô's formula, one has

$$
\begin{gathered}
\left\|u_{t}^{1, n}\right\|^{2}=2 \int_{0}^{t}\left(u_{s}^{1, n}, d u_{s}^{1, n}\right)+\int_{0}^{t}\left\|w_{s}^{1, n}\right\|_{d}^{2} d s= \\
-2 \int_{0}^{t}\left(u_{s}^{1, n}, A u_{s}^{1, n}\right) d s+2 \sum_{i=1}^{d} \int_{0}^{t}\left(u_{s}^{1, n}, w_{i, s}^{1, n}\right) d B_{s}^{i}+\int_{0}^{t}\left\|w_{s}^{1, n}\right\|_{d}^{2} d s .
\end{gathered}
$$

As for all $s \geq 0,\left(u_{s}^{1, n}, A u_{s}^{1, n}\right)=\mathcal{E}\left(u_{s}^{1, n}, u_{s}^{1, n}\right)$, we get

$$
\left\|u_{t}^{1, n}\right\|^{2}+2 \int_{0}^{t} \mathcal{E}\left(u_{s}^{1, n}\right) d s=2 \sum_{i=1}^{d} \int_{0}^{t}\left(u_{s}^{1, n}, w_{i, s}^{1, n}\right) d B_{s}^{i}+\int_{0}^{t}\left\|w_{s}^{1, n}\right\|_{d}^{2},
$$

which implies

$$
\sup _{t \leq T}\left\|u_{t}^{1, n}\right\|^{2} \leq 2 \sup _{t \leq T}\left|\sum_{i=1}^{d} \int_{0}^{t}\left(u_{s}^{1, n}, w_{i, s}^{n}\right) d B_{s}^{i}\right|+\int_{0}^{T}\left\|w_{t}^{1, n}\right\|_{d}^{2} d t .
$$

The Burkhölder-Davies-Gundy's inequality ensures that there exists a constant $c_{1}$ such that

$$
\begin{aligned}
E \sup _{t \leq T}\left|\sum_{i=1}^{d} \int_{0}^{t}\left(u_{s}^{1, n}, w_{i, s}^{1, n}\right) d B_{s}^{i}\right| & \leq c_{1} E\left(\left|\sum_{i=1}^{d} \int_{0}^{T}\left(u_{s}^{1, n}, w_{i, s}^{1, n}\right)^{2} d s\right|^{1 / 2}\right) \leq \\
& \leq c_{1} E\left(\sup _{t \leq T}\left\|u_{t}^{1, n}\right\| \times\left|\int_{0}^{T}\left\|w_{t}^{1, n}\right\|_{d}^{2} d t\right|^{1 / 2}\right) \leq \\
& \leq \varepsilon E\left(\sup _{t \leq T}\left\|u_{t}^{1, n}\right\|^{2}\right)+\frac{c_{1}^{2}}{4 \varepsilon} E\left(\int_{0}^{T}\left\|w_{t}^{1, n}\right\|_{d}^{2} d t\right),
\end{aligned}
$$


for all $\varepsilon>0$. So, taking $\varepsilon$ small enough, we get

$$
E\left(\sup _{t \leq T}\left\|u_{t}^{1, n}\right\|^{2}\right) \leq c_{2} E\left(\int_{0}^{T}\left\|w_{t}^{1, n}\right\|_{d}^{2} d t\right)
$$

where $c_{2}$ is another constant. Moreover, by taking the expectation in (6), we get

$$
E \int_{0}^{T} \mathcal{E}\left(u_{t}^{1, n}\right) d t \leq \frac{1}{2} E\left(\int_{0}^{T}\left\|w_{t}^{1, n}\right\|_{d}^{2} d t\right) .
$$

A similar calculation can be done for the difference $u^{1, n}-u^{1, m}$, so as to deduce a suitable convergence of $u^{1, n}$ to $u^{1}$ and establish the estimate

$$
E\left(\left\|u^{1}\right\|_{T}^{2}\right) \leq c E\left(\int_{0}^{T}\left\|w_{t}\right\|_{d}^{2} d t\right)
$$

with a constant $c$ which only depends on $T$. Now, one sees that $u_{t}^{n}=P_{\frac{1}{n}} u_{t}$, so as previously we may perform the following calculation

$$
u_{t}^{n}+\int_{0}^{t} A u_{s}^{n} d s=x^{n}+\sum_{i=1}^{d} \int_{0}^{t} w_{i, s}^{n} d B_{s}^{i}+\int_{0}^{t} w_{s}^{\prime, n} d s+\int_{0}^{t} z_{s}^{n} d s,
$$

which shows that $u_{t}^{n}$ is a $H$-valued semimartingale. By applying Itô's formula, one obtains

$$
\begin{gathered}
\left\|u_{t}^{n}\right\|^{2}+2 \int_{0}^{t} \mathcal{E}\left(u_{s}^{n}\right) d s=\left\|x^{n}\right\|^{2}+2 \sum_{i=1}^{d} \int_{0}^{t}\left(u_{s}^{n}, w_{i, s}^{n}\right) d B_{s}^{i}+ \\
\quad+\int_{0}^{t}\left(u_{s}^{n}, w_{s}^{\prime, n}\right) d s+\int_{0}^{t}\left(u_{s}^{n}, z_{s}^{n}\right) d s+\int_{0}^{t}\left\|w_{s}^{n}\right\|_{d}^{2} d s .
\end{gathered}
$$

Thanks to Lemmas 3,4 and the previous estimate, we know that $u_{s}^{n} \rightarrow$ $u_{s}$ in the space $L^{2}([0, t] \times \Omega ; F)$, and hence one deduces that $\left(u_{s}^{n}, z_{s}^{n}\right) \rightarrow$ $\left(u_{s}, R w_{s}^{\prime \prime}\right)_{F, F^{\prime}}$ in $L^{1}([0, t] \times \Omega ; R)$. Therefore one easily sees that the above relation passes to the limit and one gets the relation $(i i)$ of our proposition. The relation (iii) is obtained by taking the expectation.

In order to deduce the relation $(i)$, one multiplies (8) with a test function $\varphi \in \mathcal{D}$ and applies Itô's formula over the interval $[0, t]$ with $t$ such that $\varphi(t)=0$,

$$
\begin{gathered}
\int_{0}^{t}\left[\left(u_{s}^{n}, \partial_{s} \varphi\right)-\left(A u_{s}^{n}, \varphi_{s}\right)+\left(w_{s}^{\prime, n}, \varphi_{s}\right)+\left(z_{s}^{n}, \varphi_{s}\right)_{F^{\prime}, F}\right] d s \\
+\sum_{i=1}^{d} \int_{0}^{t}\left(w_{i, s}^{n}, \varphi_{s}\right) d B_{s}^{i}+\left(x^{n}, \varphi_{0}\right)=0
\end{gathered}
$$


letting $n$ tend to $\infty$, we get the relation $(i)$.

To obtain the relation (iv) of the statement, we use (7) and Lemmas 3 and 4 which ensure that, almost surely

$$
\left\|u^{2}\right\|_{T}^{2} \leq c\left(\|x\|^{2}+\int_{0}^{T}\left\|w_{t}^{\prime}\right\|^{2} d t\right),\left\|u^{3}\right\|_{T}^{2} \leq c \int_{0}^{T}\left\|w_{t}^{\prime \prime}\right\|_{K}^{2} d t .
$$

This directly leads to the desired relation.

Assume now that $w^{\prime \prime}=0$. As for all $n$

$$
u_{t}^{n}+A \int_{0}^{t} u_{s}^{n} d s=x^{n}+\sum_{i=1}^{d} \int_{0}^{t} w_{i, s}^{n} d B_{s}^{i}+\int_{0}^{t} w_{s}^{\prime, n} d s,
$$

we obtain the last assertion by letting $n$ tends to infinity, using the fact that $A$ is a closed operator.

If $f, g$ and $h$ do not depend on $u$ then the equation (2) is a linear equation. In this case the relation defining the notion of a mild solution simply becomes a relation which defines the object that is a mild solution. Proposition 6 ensures that it is also a weak solution. In general we have the following result.

Proposition 7 The notions of weak and mild solutions coincide. Any solution belongs to $\widetilde{\mathcal{P}}(F)$.

Proof. The fact that any mild solution belongs to $\widetilde{\mathcal{P}}(F)$ and is a weak solution follows from Proposition 6.

Conversely, assume that $u$ is a weak solution and define the process

$v_{t}=P_{t} x+\sum_{i=1}^{d} \int_{0}^{t} P_{t-s} h_{i}\left(s, u_{s}\right) d B_{s}^{i}-\int_{0}^{t} P_{t-s} f\left(s, u_{s}\right) d s-\int_{0}^{t} P_{t-s} R g\left(s, u_{s}\right) d s$.

We should prove that $u=v$. Comparing the value of the integral

$$
\int_{0}^{\infty}\left[\left(u_{s}, \partial_{s} \varphi\right)-\mathcal{E}\left(u_{s}, \varphi_{s}\right)\right] d s
$$

obtained from the relation defining a weak solution, and the value of the same integral with $v$ in the place of $u$, given by the relation $(i)$ of the preceding proposition, we observe that the two are almost surely equal. So, we deduce that

$$
\int_{0}^{\infty}\left[\left(u_{s}-v_{s}, \partial_{s} \varphi\right)-\mathcal{E}\left(u_{s}-v_{s}, \varphi_{s}\right)\right] d s=0,
$$

almost surely, for each $\varphi \in \mathcal{D}$. Since $\mathcal{D}$ contains a countable set which is dense in it, we deduce that the relation holds with arbitrary $\varphi \in \mathcal{D}$, outside 
of a negligeable set in $\Omega$. By Lemma 2 we deduce that $u=v$, almost surely, concluding the proof.

As the above propositions suggest, a first candidate for the space where the solutions are searched for would be $\widetilde{\mathcal{P}}(F)$. However the norms of this space are too strong and would imply strong conditions on the Lipschitz constants of the nonlinear terms to obtain a contraction inequality needed to produce the solution. To avoid this, another space will be introduced so that to obtain an existence result under relaxed conditions on the Lipschitz constants (the constants $\alpha, \beta$ and $\|R\|$ are particularly involved and the condition (H2) is the optimal choice concerning them, with respect to the contraction argument we employ). The new space is denoted by $\widehat{\mathcal{P}}(F, T)$ and consists of processes defined only on an interval $[0, T]$ of a certain length $T>$ 0 . More precisely, it is the space of all predictable processes $u:[0, T] \times \Omega \rightarrow F$ for which the norm

$$
\|u\|_{E, T}:=\left(\sup _{0 \leq t \leq T} E\left\|u_{t}\right\|^{2}+2 E \int_{0}^{T} \mathcal{E}\left(u_{t}\right) d t\right)^{1 / 2}
$$

is finite. By factorization with respect to the null sets, the pair $\left(\widehat{\mathcal{P}}(F, T),\|\|_{E, T}\right)$ becomes a Banach space.

The main existence result is the following theorem.

Theorem 8 There exists a unique solution to equation (2) with initial condition $u(0)=x \in L^{2}\left(\Omega, \mathcal{F}_{0}, P ; H\right)$ and it belongs to $\widetilde{\mathcal{P}}(F)$.

Proof. The main point in the proof will be to show that the map $\Lambda$ : $\widehat{\mathcal{P}}(F, t) \rightarrow \widehat{\mathcal{P}}(F, t)$, defined below, is a contraction with respect to the norm $\|\cdot\|_{E, t}$ for $t$ small enough:

$$
\begin{aligned}
(\Lambda u)_{t}= & P_{t} x-\int_{0}^{t} P_{t-s} f\left(s, u_{s}\right) d s-\int_{0}^{t} P_{t-s} R g\left(s, u_{s}\right) d s \\
& +\sum_{i=1}^{d} \int_{0}^{t} P_{t-s} h_{i}\left(s, u_{s}\right) d B_{s}^{i} .
\end{aligned}
$$

Proposition 6 gives, with $u, v \in \widehat{\mathcal{P}}(F, t)$,

$$
\begin{gathered}
E\left\|\Lambda u_{t}-\Lambda v_{t}\right\|^{2}+2 \int_{0}^{t} E \mathcal{E}\left(\Lambda u_{s}-\Lambda v_{s}\right) d s= \\
-2 \int_{0}^{t} E\left[\left(\Lambda u_{s}-\Lambda v_{s}, f\left(s, u_{s}\right)-f\left(s, v_{s}\right)\right)+\left(\Lambda u_{s}-\Lambda v_{s}, R\left(g\left(s, u_{s}\right)-g\left(s, v_{s}\right)\right)\right)_{F, F^{\prime}}\right] d s \\
+\int_{0}^{t} E\left\|h\left(s, u_{s}\right)-h\left(s, v_{s}\right)\right\|_{d}^{2} d s .
\end{gathered}
$$


The integrands of the three terms in the right hand side are estimated, by using the Lipschitz conditions (H1) and some elementary inequalities, as follows

$$
\begin{aligned}
& 2\left|\left(\Lambda u_{s}-\Lambda v_{s}, f\left(s, u_{s}\right)-f\left(s, v_{s}\right)\right)\right| \leq \frac{C^{2}}{\delta}\left\|\Lambda u_{s}-\Lambda v_{s}\right\|^{2}+\delta\left\|u_{s}-v_{s}\right\|_{F}^{2}, \\
& \quad 2\left|\left(\Lambda u_{s}-\Lambda v_{s}, R\left(g\left(s, u_{s}\right)-g\left(s, v_{s}\right)\right)\right)_{F, F^{\prime}}\right| \\
& \leq 2\left\|\Lambda u_{s}-\Lambda v_{s}\right\|_{F} \times\left\|R\left(g\left(s, u_{s}\right)-g\left(s, v_{s}\right)\right)\right\|_{F^{\prime}} \\
& \leq 2\left\|\Lambda u_{s}-\Lambda v_{s}\right\|_{F} \times\|R\|\left\|\left(g\left(s, u_{s}\right)-g\left(s, v_{s}\right)\right)\right\|_{K} \\
& \leq \quad 2\|R\| \times\left\|\Lambda u_{s}-\Lambda v_{s}\right\|_{F} \times\left(C\left\|u_{s}-v_{s}\right\|+\alpha \mathcal{E}\left(u_{s}-v_{s}\right)^{1 / 2}\right) \\
& \leq \quad(\delta+\alpha\|R\|)\left\|\Lambda u_{s}-\Lambda v_{s}\right\|_{F}^{2}+\|R\|^{2} C^{2} \frac{1}{\delta}\left\|u_{s}-v_{s}\right\|^{2}+\alpha\|R\| \mathcal{E}\left(u_{s}-v_{s}\right), \\
& \left\|h\left(s, u_{s}\right)-h\left(s, v_{s}\right)\right\|_{d}^{2} \leq C^{2}\left(1+\frac{1}{\delta}\right)\left\|u_{s}-v_{s}\right\|^{2}+\beta^{2}(1+\delta) \mathcal{E}\left(u_{s}-v_{s}\right) .
\end{aligned}
$$

for any $\delta>0$. Then one finds that

$$
\begin{aligned}
E \| \Lambda u_{t} & -\Lambda v_{t} \|^{2}+(2-\delta-\alpha\|R\|) E \int_{0}^{t} \mathcal{E}\left(\Lambda u_{s}-\Lambda v_{s}\right) d s \leq \\
& \left(\frac{C^{2}}{\delta}+\delta+\alpha\|R\|\right) \int_{0}^{t} E\left\|\Lambda u_{s}-\Lambda v_{s}\right\|^{2} d s \\
& +\left(\delta+C^{2}+\frac{C^{2}}{\delta}\left(\|R\|^{2}+1\right)\right) \int_{0}^{t} E\left\|u_{s}-v_{s}\right\|^{2} d s \\
& +\left(\delta+\alpha\|R\|+\beta^{2}(1+\delta)\right) E \int_{0}^{t} \mathcal{E}\left(u_{s}-v_{s}\right) d s .
\end{aligned}
$$

Now, since by the hypothesis (H2) one has $2 \alpha\|R\|+\beta^{2}<2$, we may choose two positive constants $\theta_{1}, \theta_{2}$, such that $\alpha\|R\|+\beta^{2}<\theta_{1}<\theta_{2}<2-\alpha\|R\|$. Then we take $\delta$ small enough to have $\delta+\alpha\|R\|+\beta^{2}(1+\delta)<\theta_{1}$ and $\theta_{2}<2-\delta-\alpha\|R\|$. Finally we conclude the preceding estimations obtaining

$$
\begin{gathered}
E\left\|\Lambda u_{t}-\Lambda v_{t}\right\|^{2}+\theta_{2} E \int_{0}^{t} \mathcal{E}\left(\Lambda u_{s}-\Lambda v_{s}\right) d s \leq \\
c_{3} \int_{0}^{t} E\left\|\Lambda u_{s}-\Lambda v_{s}\right\|^{2} d s+c_{4} \int_{0}^{t} E\left\|u_{s}-v_{s}\right\|^{2} d s+\theta_{1} E \int_{0}^{t} \mathcal{E}\left(u_{s}-v_{s}\right) d s,
\end{gathered}
$$

where $c_{3}$ and $c_{4}$ are constants which only depend on $C, \alpha, \beta$ and $\|R\|$. Gronwall's lemma implies that, for any $t$,

$$
E\left\|\Lambda u_{t}-\Lambda v_{t}\right\|^{2}+\theta_{2} E \int_{0}^{t} \mathcal{E}\left(\Lambda u_{s}-\Lambda v_{s}\right) d s \leq
$$




$$
e^{c_{3} t}\left[c_{4} \int_{0}^{t} E\left\|u_{s}-v_{s}\right\|^{2} d s+\theta_{1} E \int_{0}^{t} \mathcal{E}\left(u_{s}-v_{s}\right) d s\right] .
$$

The left hand side of this inequality is minorated by

$$
\frac{\theta_{2}}{2}\left(E\left\|\Lambda u_{t}-\Lambda v_{t}\right\|^{2}+2 E \int_{0}^{t} \mathcal{E}\left(\Lambda u_{s}-\Lambda v_{s}\right) d s\right)
$$

while the right hand side is dominated by

$$
e^{c_{3} t}\left(c_{4} t+\frac{\theta_{1}}{2}\right)\|u-v\|_{E, t}^{2} .
$$

Thus we get the important inequality

$$
\|\Lambda u-\Lambda v\|_{E, t}^{2} \leq \theta\|u-v\|_{E, t}^{2},
$$

with $\theta:=e^{c_{3} t}\left(c_{4} t+\frac{\theta_{1}}{2}\right)$. Choosing $t$ small enough to ensure that $\theta<1$ we obtain that $\Lambda$ is a contraction on the space $\widehat{\mathcal{P}}(F, t)$. The fixed point of this contraction $u=\Lambda u$, is a solution of the mild equation on the interval $[0, t]$. Observing that mild solutions have a flow property, one extends the solution on $\mathbf{R}_{+}$. Finally, thanks to Proposition 6 , it is clear that this solution belongs to $\widetilde{\mathcal{P}}(F)$.

The next theorem shows that the solution satisfies estimates with respect to the data similar to the estimate (iv) of Proposition 6.

Theorem 9 There exists a constant $c$ which only depends on $C, \alpha, \beta,\|R\|$ and $T$ such that the following estimate holds for the solution of the equation (2),

$$
E\|u\|_{T}^{2} \leq c E\left(\|x\|^{2}+\int_{0}^{T}\left(\|h(t, 0)\|_{d}^{2}+\|f(t, 0)\|^{2}+\|g(t, 0)\|_{K}^{2}\right) d t\right) .
$$

Proof. We shall use the inequality (9) established in the preceding proof. We first remark that the length $t$ and the constant $\theta$ have been chosen only dependent on the constants $C, \alpha, \beta$ and $\|R\|$.

A solution satisfies the relation $u=\Lambda u$ and so we may write

$$
\|u\|_{E, t}=\|\Lambda u\|_{E, t} \leq\|\Lambda u-\Lambda 0\|_{E, t}+\|\Lambda 0\|_{E, t} \leq \sqrt{\theta}\|u\|_{E, t}+\|\Lambda 0\|_{E, t},
$$

which implies the estimate

$$
\|u\|_{E, t}^{2} \leq\left(\frac{1}{1-\sqrt{\theta}}\right)^{2}\|\Lambda 0\|_{E, t}^{2} .
$$

Since in general one has $\|v\|_{E, t}^{2} \leq E\|v\|_{t}^{2}$, we may apply this and the estimate (iv) of Proposition 6 to dominate the last term of the inequality obtaining

$\|u\|_{E, t}^{2} \leq c\left(\frac{1}{1-\sqrt{\theta}}\right)^{2} E\left(\|x\|^{2}+\int_{0}^{t}\left(\|h(s, 0)\|_{d}^{2}+\|f(s, 0)\|^{2}+\|g(s, 0)\|_{K}^{2}\right) d s\right)$. 
This estimate can be iterated over each interval of the form $[n t,(n+1) t]$ so that we arrive at an estimate over an arbitrary interval $[0, T]$,

$$
\|u\|_{E, T}^{2} \leq c_{5} E\left(\|x\|^{2}+\int_{0}^{T}\left(\|h(s, 0)\|_{d}^{2}+\|f(s, 0)\|^{2}+\|g(s, 0)\|_{K}^{2}\right) d s\right),
$$

with a new constant $c_{5}$, which depends on $T$.

Now we apply the estimate (iv) of Proposition 6 to $u=\Lambda u$ obtaining

$$
E\|u\|_{T}^{2} \leq c E\left(\|x\|^{2}+\int_{0}^{T}\left(\left\|h\left(t, u_{t}\right)\right\|_{d}^{2}+\left\|f\left(t, u_{t}\right)\right\|^{2}+\left\|g\left(t, u_{t}\right)\right\|_{K}^{2}\right) d t\right) .
$$

Using the Lipschitz properties of $f, g$ and $h$ the last expression is dominated by

$$
\begin{gathered}
c_{6} E\left(\|x\|^{2}+\int_{0}^{T}\left(\|h(t, 0)\|_{d}^{2}+\|f(t, 0)\|^{2}+\|g(t, 0)\|_{K}^{2}\right) d t\right) \\
+c_{7} E \int_{0}^{T}\left(\left\|u_{t}\right\|^{2}+\mathcal{E}\left(u_{t}\right)\right) d t .
\end{gathered}
$$

Finally, since we have $E \int_{0}^{T}\left(\left\|u_{t}\right\|^{2}+\mathcal{E}\left(u_{t}\right)\right) d t \leq(1+T)\|u\|_{E, T}^{2}$, thanks to inequality (10) we obtain the estimate asserted by the theorem. $\square$

\section{Examples}

\subsection{The case of a diffusion generator in a finite-dimensional state space}

We can apply the above theorem to the standard finite dimensional diffusion operator of (1):

$$
\begin{gathered}
d u_{t}-\sum_{i, j=1}^{N} \partial_{i}\left(a^{i, j} \partial_{j} u_{t}\right) d t+\widetilde{f}\left(t, x, u_{t}, \frac{1}{\sqrt{2}} \nabla u_{t} \sigma\right) d t \\
+\sum_{i=1}^{N} \partial_{i}\left(a^{i j} \widetilde{g}_{j}\left(t, x, u_{t}, \frac{1}{\sqrt{2}} \nabla u_{t} \sigma\right)\right) d t=\sum_{i=1}^{d} \widetilde{h}_{i}\left(t, u_{t}, \frac{1}{\sqrt{2}} \nabla u_{t} \sigma\right) d B_{t}^{i} .
\end{gathered}
$$

The second order operator associated with $a$ is a semi-elliptic operator with zero Dirichlet boundary conditions. To define it precisely we follow Section 3.1 of $[9]$. So the requirements of our framework are so weak that the diffusion coefficients could be even degenerated and discontinuous. More precisely, let $D$ be a domain of the finite dimensional space $\mathbf{R}^{N}$, and $\sigma=$ $\left(\sigma_{j}^{i} / i=1, \ldots, N, j=1, \ldots, k\right)$ a measurable $(N, k)$-matrix field defined on $D$. Then the square $N$-matrix field $a=\frac{1}{2} \sigma \sigma^{*}, a^{i j}=\frac{1}{2} \sum_{l=1}^{k} \sigma_{l}^{i} \sigma_{l}^{j}$, defined 
on $D$ is measurable, symmetric and non-negative definite.We only assume that $a$ is locally integrable with respect to the Lebesgue measure and that it generates a Dirichlet space. To be more specific, let us define the bilinear form

$$
\mathcal{E}(u, v)=\int_{D} \sum_{i, i} a^{i j}(x) \partial_{i} u(x) \partial v_{j}(x) d x, \quad u, v \in \mathcal{C}_{c}^{\infty}(D)
$$

denote by $(u, v)=\int_{D} u v$ the usual scalar product in $L^{2}(D)$ and set $\mathcal{E}_{1}(u, v)=$ $\mathcal{E}(u, v)+(u, v)$. What we assume is that $\left(\mathcal{C}_{c}^{\infty}(D), \mathcal{E}_{1}\right)$ is closable in the sense of [9] (see Section 3.1). This means that the closure of $\mathcal{C}_{c}^{\infty}(D)$ with respect to the norm generated by $\mathcal{E}_{1}$ can be identified with a subspace of $L^{2}(D)$. Still following [9], we know that this is the case if $a$ is strictly elliptic or if for all $i, j \in\{1, \ldots, N\}, \frac{\partial a_{i, j}}{\partial x_{i}}$ belongs to $L_{l o c}^{2}(D)$. This last condition shows that we may consider the case where $a$ is degenerated and even the totally degenerated one, $a \equiv 0$, which corresponds to the case where we deal in fact with an (infinite dimensional) ordinary stochastic differential equation.

The basic Hilbert space $H$ is $L^{2}(D)$ and $F$ is the closure of $\mathcal{C}_{c}^{\infty}(D)$ with respect to $\mathcal{E}_{1}^{1 / 2}$. The semigroup $\left(P_{t}\right)$ is the semigroup associated to this Dirichlet space and it corresponds to zero boundary condition. The operator $A$, its infinitesimal generator, can be formally written as a divergence form operator, i. e. $A=-\sum_{i, j} \partial_{i}\left(a^{i j} \partial_{j}\right)$.

Suppose that we have the predictable functions

$$
\begin{aligned}
\widetilde{f} & : \mathbf{R}_{+} \times \Omega \times D \times \mathbf{R} \times \mathbf{R}^{k} \rightarrow \mathbf{R}, \\
\widetilde{g} & =\left(\widetilde{g}_{1}, \ldots, \widetilde{g}_{N}\right): \mathbf{R}_{+} \times \Omega \times D \times \mathbf{R} \times \mathbf{R}^{k} \rightarrow \mathbf{R}^{N}, \\
\widetilde{h} & =\left(\widetilde{h}_{1}, \ldots, \widetilde{h}_{d}\right): \mathbf{R}_{+} \times \Omega \times D \times \mathbf{R} \times \mathbf{R}^{k} \rightarrow \mathbf{R}^{d},
\end{aligned}
$$

that satisfy the Lipschitz conditions with respect to the last two variables as follows,

$$
\begin{aligned}
\left|\tilde{f}(t, \omega, x, y, z)-\tilde{f}\left(t, \omega, x, y^{\prime}, z^{\prime}\right)\right| & \leq C\left(\left|y-y^{\prime}\right|+\left|z-z^{\prime}\right|\right), \\
\left|\widetilde{g}(t, \omega, x, y, z)-\widetilde{g}\left(t, \omega, x, y^{\prime}, z^{\prime}\right)\right|_{a(x)} & \leq C\left|y-y^{\prime}\right|+\alpha\left|z-z^{\prime}\right|, \\
\left(\sum_{i=1}^{d}\left|\widetilde{h}_{i}(t, \omega, x, y, z)-\widetilde{h}_{i}\left(t, \omega, x, y^{\prime}, z^{\prime}\right)\right|^{2}\right)^{\frac{1}{2}} & \leq C\left|y-y^{\prime}\right|+\beta\left|z-z^{\prime}\right|,
\end{aligned}
$$

where $C, \alpha, \beta$ are some constants, the seminorm $|v|_{a(x)}$, for a vector $v \in \mathbf{R}^{N}$, is expressed by $|v|_{a(x)}^{2}=\sum_{i, j} a^{i j}(x) v_{i} v_{j}$ and the sign $|\cdot|$ stands for the Euclidean norm in $\mathbf{R}^{N}$ or $\mathbf{R}^{k}$. Assume also that

$$
\begin{aligned}
\tilde{f}(\cdot, \cdot, \cdot, 0,0) & \in L^{2}([0, T] \times \Omega \times D),(\forall) T>0, \\
|\widetilde{g}|_{a(\cdot)}(\cdot, \cdot, \cdot,, 0,0) & \in L^{2}([0, T] \times \Omega \times D),(\forall) T>0, \\
|\widetilde{h}|_{(\cdot, \cdot, \cdot, 0,0)} & \in L^{2}([0, T] \times \Omega \times D),(\forall) T>0 .
\end{aligned}
$$


We denote by $l_{a}^{2}$ the set of all classes of measurable functions $u: D \rightarrow \mathbf{R}^{N}$ for which the seminorm

$$
\|u\|_{L_{a}^{2}}=\left(\int_{D} \sum_{i, j} a^{i j}(x) u_{i}(x) u_{j}(x) d x\right)^{1 / 2}
$$

is finite. Then $K$ is nothing but $L_{a}^{2}$, the quotient of $l_{a}^{2}$ with respect to the subvector space $\left\{u \in l_{a}^{2},\|u\|_{L_{a}^{2}}=0\right\}$. The operator $R$ is formally defined by

$$
\forall u=\left(u_{1}, \ldots, u_{N}\right) \in L_{a}^{2}, R u=\sum_{i, j} \partial_{i}\left(a^{i j} u_{j}\right) .
$$

More precisely, as an element of the dual $F^{\prime}$ it is defined by

$$
(R u, v)_{F^{\prime}, F}=-\int_{D} \sum_{i, j} a^{i j}(x) u_{j}(x) \partial_{i} v(x) d x, \forall v \in \mathcal{C}_{c}^{\infty}(D) .
$$

For functions $v \in F$ one has a well-defined generalized gradient so that the vector $\nabla u \sigma$ makes sense $\left(\nabla u \sigma(x)\right.$ is the vector in $\mathbf{R}^{k}$ of components $\sum_{i=1}^{N}\left(\partial_{i} u\right) \sigma_{j}^{i}(x), j=1, \ldots k$; see [2] for more details) and we may write

$$
(R u, v)_{F^{\prime}, F}=-\int_{D} \frac{1}{2} \sum_{i, j} \sum_{l} \sigma_{l}^{i}(x) \sigma_{l}^{j}(x) u_{j}(x) \partial_{i} v(x) d x, \quad \forall v \in F .
$$

¿From the inequality

$$
\left|(R u, v)_{F^{\prime}, F}\right| \leq\|u\|_{L_{a}^{2}} \mathcal{E}^{1 / 2}(v)
$$

it follows that the operator norm of $R$ satisfies $\|R\| \leq 1$.

On the other hand, to the concrete functions $f, g, h$, one associates the abstract operator-functions defined on $F$,

$$
\begin{array}{ll}
f: & \mathbf{R}_{+} \times \Omega \times F \rightarrow H, f(t, \omega, u)(\cdot)=\widetilde{f}\left(t, \omega, \cdot, u(\cdot), \frac{1}{\sqrt{2}} \nabla u \sigma(\cdot)\right), \\
g: \quad & \mathbf{R}_{+} \times \Omega \times F \rightarrow K, g(t, \omega, u)(\cdot)=\widetilde{g}\left(t, \omega, \cdot, u(\cdot), \frac{1}{\sqrt{2}} \nabla u \sigma(\cdot)\right), \\
h \quad: \quad \mathbf{R}_{+} \times \Omega \times F \rightarrow H^{d}, h(t, \omega, u)(\cdot)=\widetilde{h}\left(t, \omega, \cdot, u(\cdot), \frac{1}{\sqrt{2}} \nabla u \sigma(\cdot)\right) .
\end{array}
$$

The Lipschitz condition of $g$ implies that for $u, v \in F$ one has

$$
\begin{gathered}
\|g(t, \omega, u)-g(t, \omega, v)\|_{K} \\
=\left\|\widetilde{g}\left(t, \omega, \cdot, u(\cdot), \frac{1}{\sqrt{2}} \nabla u \sigma(\cdot)\right)-\widetilde{g}\left(t, \omega, \cdot, v(\cdot), \frac{1}{\sqrt{2}} \nabla v \sigma(\cdot)\right)\right\|_{L_{a}^{2}}
\end{gathered}
$$




$$
\begin{gathered}
=\left\|\left|\widetilde{g}\left(t, \omega, \cdot, u(\cdot), \frac{1}{\sqrt{2}} \nabla u \sigma(\cdot)\right)-\widetilde{g}\left(t, \omega, \cdot, v(\cdot), \frac{1}{\sqrt{2}} \nabla v \sigma(\cdot)\right)\right|_{a}\right\|_{L^{2}} \\
\leq\left\|C|u-v|+\alpha\left|\frac{1}{\sqrt{2}}(\nabla u-\nabla v) \sigma\right|\right\|_{L^{2}} \leq C\|u-v\|_{L^{2}}+\alpha\left\|\left|\frac{1}{\sqrt{2}}(\nabla u-\nabla v) \sigma\right|\right\|_{L^{2}} \\
=C\|u-v\|_{L^{2}}+\alpha \mathcal{E}^{1 / 2}(u-v) .
\end{gathered}
$$

This shows that the condition in (H1) is fulfilled. Similarly one can verify the other conditions in (H1) concerning $f$ and $h$. In the present case the condition (H2) simply becomes $2 \alpha+\beta^{2}<2$ and the condition (H3) immediately follows from the assumption $\left(H^{\prime}\right)$. Thus we deduce from our main theorem that under the condition $2 \alpha+\beta^{2}<2$ the equation (1) has a unique solution and it satisfies certain estimates. Under uniform ellipticity we will prove a double stochastic formula in the last section.

\subsection{An example with the bi-laplacian}

We consider the following equation:

$$
\begin{gathered}
d u_{t}+\Delta^{2} u_{t} d t+\widetilde{f}\left(t, x, u_{t}, \nabla u_{t}, D^{2} u_{t}\right) d t \\
+\Delta \widetilde{g}\left(t, x, u_{t}, \nabla u_{t}, D^{2} u_{t}\right) d t=\sum_{i=1}^{d} \widetilde{h}_{i}\left(t, x, u_{t}, \nabla u_{t}, D^{2} u_{t}\right) d B_{t}^{i},
\end{gathered}
$$

with initial condition $u_{0} \in L^{2}\left(\mathbf{R}^{N}\right)$, where the state space is $\mathbf{R}^{N}, \nabla u=$ $\left(\partial_{1} u, \ldots \partial_{N} u\right)$ is the gradient, $D^{2} u=\left(\partial_{i} \partial_{j} u, i, j=1, \ldots N\right)$ is the Hessian of a function $u$ and $\Delta$ is the Laplace operator. The functions $\tilde{f}, \widetilde{g}, \widetilde{h}$ are defined as follows

$$
\begin{aligned}
\widetilde{f}, \widetilde{g} & : \quad \mathbf{R}_{+} \times \Omega \times \mathbf{R}^{N} \times \mathbf{R} \times \mathbf{R}^{N} \times \mathbf{R}^{N \times N} \rightarrow \mathbf{R} \\
\widetilde{h}= & \left(\widetilde{h}_{1}, \cdots, \widetilde{h}_{d}\right): \mathbf{R}_{+} \times \Omega \times \mathbf{R}^{N} \times \mathbf{R} \times \mathbf{R}^{N} \times \mathbf{R}^{N \times N} \rightarrow \mathbf{R}^{d} .
\end{aligned}
$$

They are assumed to be predictable, uniformly Lipschitz continuous with respect to the last three variables, $\widetilde{f}(\cdot, \cdot, \cdot, 0,0,0), \widetilde{g}(\cdot, \cdot, \cdot, 0,0,0), \widetilde{h}_{i}(\cdot, \cdot, \cdot, 0,0,0) \in$ $L^{2}\left([0, T] \times \Omega \times \mathbf{R}^{N}\right)$ for any $T>0$, and $i \in\{1, \cdots, d\}$. More precisely this continuity is expressed by

$$
\begin{aligned}
\left|\widetilde{f}(t, \omega, x, y, z, w)-\widetilde{f}\left(t, \omega, x, y^{\prime}, z^{\prime}, w^{\prime}\right)\right| & \leq C\left(\left|y-y^{\prime}\right|+\left|z-z^{\prime}\right|+\left|w-w^{\prime}\right|\right), \\
\left|\widetilde{g}(t, \omega, x, y, z, w)-\widetilde{g}\left(t, \omega, x, y^{\prime}, z^{\prime}, w^{\prime}\right)\right| & \leq C\left(\left|y-y^{\prime}\right|+\left|z-z^{\prime}\right|\right)+\alpha\left|w-w^{\prime}\right|, \\
\sqrt{\sum_{i=1}^{d}\left|\widetilde{h}_{i}(t, \omega, x, y, z, w)-\widetilde{h}_{i}(t, \omega, x, y, z, w)\right|^{2}} & \leq C\left(\left|y-y^{\prime}\right|+\left|z-z^{\prime}\right|\right)+\beta\left|w-w^{\prime}\right|, \\
t \in \mathbf{R}_{+}, \omega \in \Omega, x \in \mathbf{R}^{N}, y, y^{\prime} \in \mathbf{R}, z, z^{\prime} & \in \mathbf{R}^{N}, w, w^{\prime} \in \mathbf{R}^{N \times N} .
\end{aligned}
$$


The constant $C$ is arbitrary while $\alpha$ and $\beta$ should satisfy the inequality $2 \alpha N^{2}+\beta^{2} N^{4}<2$, which ensures the fulfillment of the technical condition (H2). The bi-laplacian $\Delta^{2}$ is simply the square of the standard Laplacian on $R^{N}$. So one has

$$
H=K=L^{2}\left(\mathbf{R}^{N}\right), A=\Delta^{2}, F=J=\mathcal{D}(\Delta)=W^{2,2}\left(\mathbf{R}^{N}\right), R=\Delta .
$$

The "concrete" functions $\widetilde{f}, \widetilde{g}, \widetilde{h}$ of this example yield the following functions acting on the functional spaces,

$$
\begin{aligned}
f(t, \omega, u) & =\widetilde{f}\left(t, \omega, \cdot, u, \nabla u, D^{2} u\right), g(t, \omega, u)=\widetilde{g}\left(t, \omega, \cdot, u, \nabla u, D^{2} u\right), \\
h(t, \omega, u) & =\widetilde{h}\left(t, \omega, \cdot, u, \nabla u, D^{2} u\right),
\end{aligned}
$$

where $f, g: \mathbf{R}_{+} \times F \rightarrow H$ and $h: \mathbf{R}_{+} \times F \rightarrow H^{d}$. In order to check the condition (H2) of the abstract framework, we use the following inequalities

$$
\begin{aligned}
\left\|\partial_{i} \partial_{j} u\right\| & \leq\|\Delta u\|, u \in W^{2,2}\left(\mathbf{R}^{N}\right), \\
\|\nabla u\| & =\sqrt{-(u, \Delta u)} \leq \varepsilon\|\Delta u\|+C_{\varepsilon}\|u\|, u \in W^{2,2}\left(\mathbf{R}^{N}\right),
\end{aligned}
$$

with $\varepsilon$ arbitrary small and $C_{\varepsilon}$ depending on $\varepsilon$. So, one has

$$
\begin{aligned}
\|g(t, \omega, u)-g(t, \omega, v)\| & \leq C(\|u-v\|+\|\nabla u-\nabla v\|)+\alpha\left\|D^{2}(u-v) \mid\right\| \\
& \leq C_{\varepsilon}\|u-v\|+\left(\varepsilon+\alpha N^{2}\right)\|\Delta(u-v)\| \\
& =C_{\varepsilon}\|u-v\|+\left(\varepsilon+\alpha N^{2}\right) \mathcal{E}(u-v)^{1 / 2}
\end{aligned}
$$

and a similar relation for $h$. Moreover, it is clear that in this case, $\|R\| \leq 1$.

The s.p.d.e. associated to the bi-laplacian models some physical phenomena (see [4] and the references therein for further results).

\subsection{An example with an infinite dimensional state space}

We use the notation of [3] and denote by $(W, m)$ the Wiener space with Wiener measure on it, $\mathcal{H}$ denotes the Cameron-Martin space, $D$ is the Malliavin derivative, $\delta$ is its adjoint, the divergence. The s.p.d.e. we consider has the form

$$
\begin{aligned}
& d u_{t}-\frac{1}{2} \delta D u_{t} d t+\widetilde{f}\left(t, \omega, w, u_{t}, D u_{t}\right) d t+\delta \widetilde{g}\left(t, \omega, w, u_{t}, D u_{t}\right) d t \\
= & \sum_{i=1}^{d} \widetilde{h}_{i}\left(t, \omega, w, u_{t}, D u_{t}\right) d B_{t}^{i}(\omega) .
\end{aligned}
$$

The functions $\widetilde{f}, \widetilde{g}, \widetilde{h}$ are defined as follows

$$
\begin{aligned}
\tilde{f}: & R_{+} \times \Omega \times W \times R \times \mathcal{H} \rightarrow R, \\
\widetilde{g}: & R_{+} \times \Omega \times W \times R \times \mathcal{H} \rightarrow \mathcal{H}, \\
\widetilde{h} & =\left(\widetilde{h}_{1}, \cdots, \widetilde{h}_{d}\right): R_{+} \times \Omega \times W \times R \times \mathcal{H} \rightarrow R^{d} .
\end{aligned}
$$


They are predictable, satisfy the integrability conditions

$$
\begin{aligned}
f(\cdot, \cdot, \cdot, 0,0), h_{i}(\cdot, \cdot, \cdot, 0,0) & \in L^{2}([0, t] \times \Omega \times W), t>0, i \in\{1, \cdots, d\} \\
g(\cdot, \cdot, \cdot, 0,0) & \in L^{2}([0, t] \times \Omega \times W ; \mathcal{H}), t>0
\end{aligned}
$$

and the Lipschitz condition in the form

$$
\begin{aligned}
\left|\tilde{f}(t, \omega, w, y, z)-\widetilde{f}\left(t, \omega, w, y^{\prime}, z^{\prime}\right)\right| & \leq C\left(\left|y-y^{\prime}\right|+\left|z-z^{\prime}\right|_{\mathcal{H}}\right), \\
\left|\widetilde{g}(t, \omega, w, y, z)-\widetilde{g}\left(t, \omega, w, y^{\prime}, z^{\prime}\right)\right|_{\mathcal{H}} & \leq C\left|y-y^{\prime}\right|+\alpha\left|z-z^{\prime}\right|_{\mathcal{H}}, \\
\sqrt{\sum_{i=1}^{d}\left|\widetilde{h}_{i}(t, \omega, w, y, z)-\widetilde{h}_{i}\left(t, \omega, w, y^{\prime}, z^{\prime}\right)\right|^{2}} & \leq C\left|y-y^{\prime}\right|+\beta\left|z-z^{\prime}\right|_{\mathcal{H}},
\end{aligned}
$$

with an arbitrary constant $C$ and $\alpha, \beta$ satisfying $2 \alpha+\beta^{2}<1$.

In this case the state space is $W$ and $H=L^{2}(W, m), A=-\frac{1}{2} \delta D$ is the infinite dimensional Ornstein-Uhlenbeck operator, $F=\mathbb{D}^{1,2}$, and $\mathcal{E}(u, v)=\frac{1}{2} \int\langle u, v\rangle_{\mathcal{H}} d m$. The semigroup $\left(P_{t}\right)$ generated by $A$ is the infinite dimensional Ornstein-Uhlenbeck semigroup. The space $K$ is $L^{2}(W, m ; \mathcal{H})$, while $R=\delta$. A direct verification shows that the conditions imposed on $\alpha$ and $\beta$ ensures applicability of the general result.

\section{Doubly stochastic interpretation of solutions}

The doubly stochastic interpretation of solutions of s.p.d.e. was first explored in [15] and turned out to be a powerful tool, giving a direct approach to the study of s.p.d.e.'s. Further recent investigations are in [1] and [17]. In this section we only show that the doubly stochastic interpretation holds in our case too.

We first construct an approximating sequence for the solution of the following simple form of the main equation

$$
d u_{t}+A u_{t} d t=\sum_{j=1}^{d} w_{j, t} d B_{t}^{j}, u_{0}=0
$$

We do this in the abstract setting. In fact, we already know an expression for the solution and it was studied in Proposition 6. It will be approximated by solutions of the following deterministic equation with suitable data $w^{n}$,

$$
\left(\partial_{t}+A\right) v^{n}=w^{n}, v_{0}^{n}=0 .
$$

The next lemma may presumably have other applications. We are not aware wether it is known. 
Lemma 10 Let $w=\left(w_{1}, \ldots, w_{d}\right): \mathbf{R}_{+} \times \Omega \rightarrow(\mathcal{D}(A))^{d}$, be such that $w \in$ $(\widetilde{\mathcal{P}}(F))^{d}$ and

$$
E \sup _{t \leq T}\left\|A w_{t}\right\|_{d}^{2}<\infty, E \sup _{t \leq T}\left\|w_{t}\right\|_{d}^{4}<\infty,(\forall) T>0 .
$$

Define the following process in the sense of Proposition 6,

$$
u_{t}=\sum_{j=1}^{d} \int_{0}^{t} P_{t-s} w_{j, s} d B_{s}^{j}
$$

and set

$$
v_{t}^{n}=\int_{0}^{t} P_{t-s} w_{s}^{n} d s
$$

where the integrand in the right hand side is defined by $w_{t}^{n}=\sum_{j=1}^{d} 2^{n}\left(B_{t_{i+1}^{n}}^{j}-B_{t_{i}^{n}}^{j}\right) w_{j, t_{i}}$ for $t \in\left[t_{i}^{n}, t_{i+1}^{n}\right)$ and $t_{i}^{n}=\frac{i}{2^{n}}, i \in N$.

Then $v^{n}$ converges to $u$, in the following sense

$$
\lim _{n} E \sup _{t \leq T}\left\|v_{t}^{n}-u_{t}\right\|^{2}=0,(\forall) T>0
$$

As a consequence, there exists a subsequence $\left(v^{n_{l}}\right)_{l \in N}$ such that

$$
\limsup _{l}\left\|v_{t}^{n_{l}}-u_{t}\right\|=0, \text { a.s. }(\forall) T>0 .
$$

Proof. We are going to compare the approximands $v^{n}$ with the processes $u^{n}$, defined by

$$
u_{t}^{n}=\sum_{j=1}^{d} \int_{0}^{t} P_{t-s} \hat{w}_{j, s}^{n} d B_{s}^{j}, \hat{w}_{j, s}^{n}=w_{j, t_{i}^{n}}, s \in\left(t_{i}^{n}, t_{i+1}^{n}\right] .
$$

By Proposition 6 , we have

$$
\lim _{n \rightarrow+\infty} E \sup _{t \leq T}\left\|u_{t}-u_{t}^{n}\right\|^{2}=0 .
$$

We are first going to estimate $u_{\tau}^{n}-v_{\tau}^{n}$ for some fixed dyadic number $\tau$ which is written as $\frac{k_{n}}{2^{n}}$ for any large enough $n$, that we also considered as fixed for the moment. One starts with the remark that we may write the value of $v_{\tau}^{n}$ in the form of a stochastic integral,

$$
v_{\tau}^{n}=\sum_{j=1}^{d} \int_{0}^{\tau} e_{j, s}^{n} d B_{s}^{j}
$$


where $e_{j, s}^{n}=2^{n} \int_{t_{i}^{n}}^{t_{i+1}^{n}} P_{\tau-r} w_{j, t_{i}} d r$, for $s \in\left[t_{i}^{n}, t_{i+1}^{n}\right)$ with $t_{i+1}^{n} \leq \tau$ and $j \in$ $\{1, \ldots, d\}$; then we may write

$$
E\left\|u_{\tau}^{n}-v_{\tau}^{n}\right\|^{2}=\sum_{j=1}^{d} E \int_{0}^{\tau}\left\|P_{\tau-s} \hat{w}_{j, s}^{n}-e_{j, s}^{n}\right\|^{2} d s .
$$

Now, considering $j \in\{1, \ldots, d\}$ and $s \in\left[t_{i}^{n}, t_{i+1}^{n}\right)$ with $t_{i+1}^{n} \leq \tau$,we have

$P_{\tau-s} \hat{w}_{j, s}^{n}-e_{j, s}^{n}=P_{\tau-s} w_{j, t_{i}^{n}}-2^{n} \int_{t_{i}^{n}}^{t_{i+1}^{n}} P_{\tau-r} w_{j, t_{i}^{n}} d r=2^{n} \int_{t_{i}^{n}}^{t_{i+1}^{n}}\left(P_{\tau-s} w_{j, t_{i}^{n}}-P_{\tau-r} w_{j, t_{i}^{n}}\right) d r$.

Then, using the estimate $\left\|P_{t} x-x\right\| \leq t\|A x\|$, we get

$$
\left\|P_{\tau-s} \hat{w}_{j, s}^{n}-e_{j, s}^{n}\right\| \leq 2^{n} \int_{t_{i}^{n}}^{t_{i+1}^{n}}|r-s|\left\|A w_{j, t_{i}^{n}}\right\| d s \leq \frac{1}{2^{n}}\left\|A w_{j, t_{i}^{n}}\right\|,
$$

which yields

$$
E\left\|u_{\tau}^{n}-v_{\tau}^{n}\right\|^{2} \leq\left(\frac{1}{2^{n}}\right)^{2} \tau E \sup _{t \leq \tau}\left\|A w_{t}\right\|_{d}^{2} .
$$

Using this estimate for all dyadic numbers less than $\tau$, we get

$$
E \sum_{i=0}^{2^{n} \tau}\left\|u_{t_{i}^{n}}^{n}-v_{t_{i}^{n}}^{n}\right\|^{2} \leq \frac{1}{2^{n}} C_{\tau}
$$

where $C_{\tau}$ is a constant which depends only on $\tau$.

Now we are going to estimate the oscillation of $u^{n}$ with respect to our partition. Thus, for $t \in\left(t_{i}^{n}, t_{i+1}^{n}\right)$ and $t_{i+1}^{n} \leq \tau$ we have thanks to Proposition 6 ,

$$
u_{t}^{n}-u_{t_{i}^{n}}^{n}=-\int_{t_{i}^{n}}^{t} A u_{s}^{n} d s+\sum_{j=1}^{d} \int_{t_{i}^{n}}^{t} \hat{w}_{j, s}^{n} d B_{s}^{j},
$$

which leads to

$$
\begin{gathered}
\sup _{t \in\left(t_{i}^{n}, t_{i+1}^{n}\right)}\left\|u_{t}^{n}-u_{t_{i}^{n}}^{n}\right\|^{2} \leq \\
\leq(d+1)\left(\frac{1}{2^{n}}\left(\int_{t_{i}^{n}}^{t_{i+1}^{n}}\left\|A u_{s}^{n}\right\|^{2} d s\right)+\sum_{j=1}^{d}\left\|w_{j, t_{i}^{n}}\right\|^{2} \sup _{t \in\left(t_{i}^{n}, t_{i+1}^{n}\right)}\left(B_{t}^{j}-B_{t_{i}^{n}}^{j}\right)^{2}\right),
\end{gathered}
$$

and hence

$$
\sup _{i \in\left\{0, . ., 2^{n} \tau-1\right\}} \sup _{t \in\left(t_{i}^{n}, t_{i+1}^{n}\right)}\left\|u_{t}^{n}-u_{t_{i}^{n}}^{n}\right\|^{2} \leq(d+1)\left(\frac{1}{2^{n}} X^{n}+U^{n}\right),
$$

where

$$
X^{n}=\int_{0}^{\tau}\left\|A u_{s}^{n}\right\|^{2} d s
$$


and

$$
U^{n}=\sup _{i \in\left\{0, \ldots, 2^{n} \tau-1\right\}} \sum_{j=1}^{d}\left\|w_{j, t_{i}^{n}}\right\|^{2} \sup _{t \in\left(t_{i}^{n}, t_{i+1}^{n}\right)}\left(B_{t}^{j}-B_{t_{i}^{n}}^{j}\right)^{2}
$$

As

$$
\forall s \in[0, \tau], A u_{s}^{n}=\sum_{j=1}^{d} \int_{0}^{s} P_{s-r} A \widehat{w}_{j, r}^{n} d B_{r}^{j},
$$

we get using again Proposition 6

$$
E X^{n} \leq \tau E \int_{0}^{\tau}\left\|A \widehat{w}_{r}^{n}\right\|_{d}^{2} d r \leq \tau^{2} E \sup _{t \in[0, \tau]}\left\|A w_{t}\right\|_{d}^{2} .
$$

The dominated convergence theorem ensures that

$$
\lim _{n \rightarrow+\infty} E U^{n}=0
$$

and so we conclude from the preceding estimates

$$
\lim _{n \rightarrow+\infty} E\left(\sup _{i \in\left\{0, . ., 2^{n} \tau-1\right\}} \sup _{t \in\left(t_{i}^{n}, t_{i+1}^{n}\right)}\left\|u_{t}^{n}-u_{t_{i}^{n}}^{n}\right\|^{2}\right)=0 .
$$

Further we are similarly going to estimate the oscillation of $v^{n}$. Again with $t \in\left(t_{i}^{n}, t_{i+1}^{n}\right)$ and $t_{i+1}^{n} \leq \tau$,

$$
v_{t}^{n}=P_{t-t_{i}^{n}} v_{t_{i}^{n}}^{n}+\sum_{j=1}^{d} 2^{n}\left(B_{t_{i+1}^{n}}^{j}-B_{t_{i}^{n}}^{j}\right) \int_{t_{i}^{n}}^{t} P_{t-s} w_{j, t_{i}^{n}} d s
$$

and hence

$$
\left\|v_{t}^{n}-v_{t_{i}^{n}}^{n}\right\|^{2} \leq(d+1)\left(\left\|P_{t-t_{i}^{n}} v_{t_{i}^{n}}^{n}-v_{t_{i}^{n}}^{n}\right\|^{2}+\sum_{j=1}^{d}\left(B_{t_{i+1}^{n}}^{j}-B_{t_{i}^{n}}^{j}\right)^{2}\left\|2^{n} \int_{t_{i}^{n}}^{t} P_{t-s} w_{j, t_{i}^{n}} d s\right\|^{2}\right) .
$$

In order to estimate this, one takes into account the following

$$
\begin{gathered}
\left\|P_{t-t_{i}^{n}} v_{t_{i}^{n}}^{n}-v_{t_{i}^{n}}^{n}\right\|^{2} \leq\left(\frac{1}{2^{n}}\right)^{2}\left\|A v_{t_{i}^{n}}^{n}\right\|^{2}, \\
\left\|2^{n} \int_{t_{i}^{n}}^{t} P_{t-s} w_{j, t_{i}^{n}} d s\right\|^{2} \leq\left\|w_{j, t_{i}^{n}}\right\|^{2} .
\end{gathered}
$$

So we get

$$
\sup _{i \in\left\{0, . ., 2^{n} \tau-1\right\}} \sup _{t \in\left(t_{i}^{n}, t_{i+1}^{n}\right)}\left\|v_{t}^{n}-v_{t_{i}^{n}}^{n}\right\|^{2} \leq(d+1)\left(\frac{1}{2^{2 n}} V^{n}+Y^{n}\right),
$$


with

$$
V^{n}=\sup _{i \in\left\{0, \ldots, 2^{n} \tau-1\right\}}\left\|A v_{t_{i}^{n}}^{n}\right\|^{2}
$$

and

$$
Y^{n}=\sup _{i \in\left\{0, \ldots, 2^{n} \tau-1\right\}} \sum_{j=1}^{d}\left(B_{t_{i+1}^{n}}^{j}-B_{t_{i}^{n}}^{j}\right)^{2}\left\|w_{j, t_{i}^{n}}\right\|^{2} .
$$

The conditions imposed to $w$ and the dominated convergence theorem lead to $\lim _{n \rightarrow+\infty} E Y^{n}=0$. On the other hand we have $A v_{t_{i}^{n}}^{n}=\sum_{j=1}^{d} \int_{0}^{t_{i}^{n}} A e_{j, s}^{n} d B_{s}^{j}$, which implies via Doob's inequality,

$$
E \sup _{i \in\left\{0, \ldots, 2^{n} \tau\right\}}\left\|A v_{t_{i}^{n}}^{n}\right\|^{2} \leq 4 E \int_{0}^{\tau}\left\|A e_{s}^{n}\right\|_{d}^{2} d s \leq 4 \tau E \sup _{t \in[0, \tau]}\left\|A w_{t}\right\|_{d}^{2},
$$

because if $s \in\left[t_{i}^{n}, t_{i+1}^{n}\left[, A e_{j, s}^{n}=2^{n} \int_{t_{i}^{n}}^{t_{n+1}^{n}} P_{\tau-r} A w_{j, t_{i}^{n}} d r\right.\right.$. Therefore we deduce that

$$
\lim _{n \rightarrow \infty} E\left(\sup _{i \in\left\{0, . ., 2^{n} \tau-1\right\}} \sup _{t \in\left(t_{i}^{n}, t_{i+1}^{n}\right)}\left\|v_{t}^{n}-v_{t_{i}^{n}}^{n}\right\|^{2}\right)=0 .
$$

It is now easy to conclude from this relation, (11) and (12).

Now we are going to apply the preceding results to a more concrete object, which is a particular case of the first example from the introduction. Namely we shall assume that $H=L^{2}\left(\mathbf{R}^{N}\right)$ and $A=-L=-\sum_{i, j} \partial_{i}\left(a^{i j} \partial_{j}\right)$ is the infinitesimal generator of a symmetric diffusion process in $\mathbf{R}^{N}$ with measurable coefficients satisfying the uniform ellipticity condition,

$$
\lambda|\xi|^{2} \leq \sum_{i, j} a^{i j}(x) \xi^{i} \xi^{j} \leq \Lambda|\xi|^{2}, x, \xi \in \mathbf{R}^{N},
$$

where $\lambda$ and $\Lambda$ are positive constants. This means that $F$ coincides with the Sobolev space $W_{0}^{1,2}\left(\mathbf{R}^{N}\right)=W^{1,2}\left(\mathbf{R}^{N}\right)$. When the diffusion is obtained as the solution of a stochastic differential equation, then the diffusion coefficients are expressed in terms of the coefficients of the stochastic differential equation, which are usually denoted by $\sigma_{k}^{i}$, as follows

$$
a^{i j}=\frac{1}{2} \sum_{k=1}^{n} \sigma_{k}^{i} \sigma_{k}^{j}
$$

where $n$ represents the dimension of the Wiener process driving the stochastic differential equation. Because it is common to write the backward stochastic differential equations with this notation, and this is what we will do next, we will preserve the notation. However we will not use the underlying probability space of the Wiener process, rather we work with the Markov realization of the diffusion, $X=\left(\Omega^{\prime}, \mathcal{F}^{\prime}, \mathcal{F}_{t}^{\prime}, X_{t}, \theta_{t}, P^{x}\right)$, on the 
canonical space of trajectories $\Omega^{\prime}=\mathcal{C}\left([0, \infty) ; \mathbf{R}^{N}\right)$. The martingale part of the component $X_{t}^{i}$ will be denoted $M^{i}$, while the backward martingale part will be denoted by $\overleftarrow{M}^{i}$. For these notions, we refer to [9], p 246; this is a particular case of the theory of symmetric Markov processes associated to (symmetric) Dirichlet forms. The Lebesgue measure in $\mathbf{R}^{N}$ will be denoted $m$ and $T>0$ will be a fixed number. Still following [9], we know that

$$
\left\langle M^{i}, M^{j}\right\rangle_{t}=2 \int_{0}^{t} a^{i j}\left(X_{s}\right) d s
$$

and that for all $u \in F$, the process $t \rightarrow u\left(X_{t}\right)$ admits a continuous version.

Proposition 11 If $w=\left(w_{1}, \ldots, w_{d}\right) \in\left(\mathcal{P}\left(L^{2}\left(R^{N}\right)\right)\right)^{d}$, then the process $u_{t}=\sum_{j=1}^{d} \int_{0}^{t} P_{t-s} w_{j, s} d B_{s}^{j}$ admits a double stochastic representation as follows,

$$
\begin{aligned}
u\left(\omega, t, X_{T-t}\left(\omega^{\prime}\right)\right)= & \sum_{j=1}^{d} \int_{0}^{t} w_{j}\left(\omega, s, X_{T-s}\left(\omega^{\prime}\right)\right) d B_{s}^{j}(\omega)- \\
& -\sum_{i=1}^{N} \int_{T-t}^{T} \partial_{i} u\left(\omega, T-s, X_{s}\left(\omega^{\prime}\right)\right) d M_{s}^{i}\left(\omega^{\prime}\right) .
\end{aligned}
$$

The relation holds $P \otimes P^{m}$-almost surely, for each $0 \leq t \leq T$.

Proof. We first establish the formula in the case of an elementary process,

$$
w_{j}(\omega, t, x)=\chi_{\Lambda j}(\omega) \chi_{\left(r_{1}, r_{2}\right]}(t) e_{j}(x),
$$

with $0 \leq r_{1} \leq r_{2}$ and for all $j \in\{1, \ldots, d\}, \Lambda_{j} \in \mathcal{F}_{r_{1}}, e_{j} \in \mathcal{D}\left(A^{3 / 2}\right) \cap$ $L^{\infty}\left(R^{N}\right)$ so that $A e_{j} \in F$. We recall that $\mathcal{D}\left(A^{3 / 2}\right) \cap L^{\infty}\left(R^{N}\right)$ is dense in $L^{2}\left(R^{N}\right)$ (see [3] or [9]). In this case we may apply the previous lemma with the same notations in the space $L^{2}\left(R^{N}\right)$ as well as in $F$ so that besides the relations from the lemma, we get

$$
\lim _{n} E \sup _{t \leq T} \mathcal{E}\left(v_{t}^{n}-u_{t}\right)=0
$$

Since one has $\left(\partial_{t}+A\right) v^{n}=w^{n}$, for each point $\omega \in \Omega$, we may write the following relation, $P^{m}\left(d \omega^{\prime}\right)$-almost surely (see [2]),

$$
\begin{aligned}
v^{n}\left(\omega, t, X_{T-t}\left(\omega^{\prime}\right)\right)= & \int_{0}^{t} w^{n}\left(\omega, s, X_{T-s}\left(\omega^{\prime}\right)\right) d s \\
& -\sum_{i=1}^{N} \int_{T-t}^{T} \partial_{i} v^{n}\left(\omega, T-s, X_{s}\left(\omega^{\prime}\right)\right) d M_{s}^{i}\left(\omega^{\prime}\right) .
\end{aligned}
$$


Taking the limit with $n \rightarrow \infty$ in this relation, we get the formula of the statement. Convergence of the martingale term follows from relation (13), because

$$
\begin{gathered}
E^{m}\left(\sum_{i=1}^{N} \int_{T-t}^{T}\left(\partial_{i} v^{n}\left(\omega, T-s, X_{s}\left(\omega^{\prime}\right)\right)-\partial_{i} u\left(\omega, T-s, X_{s}\left(\omega^{\prime}\right)\right)\right) d M_{s}^{i}\left(\omega^{\prime}\right)\right)^{2}= \\
E^{m} \sum_{i, j=1}^{N} \int_{T-t}^{T} a^{i, j}\left(X_{s}\left(\omega^{\prime}\right)\right)\left(\partial_{i}\left(v^{n}-u\right) \partial_{j}\left(v^{n}-u\right)\right)\left(\omega, T-s, X_{s}\left(\omega^{\prime}\right)\right) d s= \\
=\int_{T-t}^{T} \mathcal{E}\left(\left(v^{n}-u\right)(\omega, T-s)\right) d s,
\end{gathered}
$$

where $E^{m}$ denotes the expectation under $P^{m}$. We should only see what happens with the first term in the right hand side of the relation (14). We shall take, as in the previous proof with the same notations, a fixed dyadic number $\tau$. We have

$$
\begin{gathered}
\int_{0}^{\tau} w^{n}\left(\omega, s, X_{T-s}\left(\omega^{\prime}\right)\right) d s \\
=\sum_{i=0}^{2^{n} \tau-1} 2^{n} \sum_{j=1}^{d}\left(B_{t_{i+1}^{n}}^{j}-B_{t_{i}^{n}}^{j}\right) \int_{t_{i}^{n}}^{t_{i+1}^{n}} w_{j}\left(\omega, t_{i}^{n}, X_{T-s}\left(\omega^{\prime}\right)\right) d s .
\end{gathered}
$$

On the other hand, the integral

$$
\int_{0}^{\tau} w_{j}\left(\omega, s, X_{T-s}\left(\omega^{\prime}\right)\right) d B_{s}^{j}(\omega)
$$

is approximated in the usual way by

$$
\sum_{i=0}^{2^{n} \tau-1}\left(B_{t_{i+1}^{n}}^{j}(\omega)-B_{t_{i}^{n}}^{j}(\omega)\right) w_{j}\left(\omega, t_{i}^{n}, X_{T-t_{i}^{n}}\left(\omega^{\prime}\right)\right)
$$

To see that (15) converges to (16) one should estimate the following

$$
d^{n}=\sum_{i=0}^{2^{n} \tau-1} 2^{n} \sum_{j=1}^{d}\left(B_{t_{i+1}^{n}}^{j}-B_{t_{i}^{n}}^{j}\right) \int_{t_{i}^{n}}^{t_{i+1}^{n}}\left[w_{j}\left(\omega, t_{i}^{n}, X_{T-s}\left(\omega^{\prime}\right)\right)-w_{j}\left(\omega, t_{i}^{n}, X_{T-t_{i}^{n}}\left(\omega^{\prime}\right)\right)\right] d s .
$$

Taking the expectation with respect to $P(d \omega)$, on has

$$
\begin{aligned}
E\left(d^{n}\right)^{2} & =E \sum_{i=0}^{2^{n} \tau-1} 2^{n} \sum_{j=1}^{d}\left(\int_{t_{i}^{n}}^{t_{i+1}^{n}}\left[w_{j}\left(\omega, t_{i}^{n}, X_{T-s}\left(\omega^{\prime}\right)\right)-w_{j}\left(\omega, t_{i}^{n}, X_{T-t_{i}^{n}}\left(\omega^{\prime}\right)\right)\right] d s\right)^{2} \\
& \leq E \sum_{i=0}^{2^{n} \tau-1} \sum_{j=1}^{d} \int_{t_{i}^{n}}^{t_{i+1}^{n}}\left(w_{j}\left(\omega, t_{i}^{n}, X_{T-s}\left(\omega^{\prime}\right)\right)-w_{j}\left(\omega, t_{i}^{n}, X_{T-t_{i}^{n}}\left(\omega^{\prime}\right)\right)\right)^{2} d s .
\end{aligned}
$$


Now, we use the explicit formula for $w$, so that for $n$ large enough

$$
\begin{aligned}
E\left(d^{n}\right)^{2} & \leq \sum_{j=1}^{d} P\left(\Lambda_{j}\right) \sum_{i=2^{n}\left(\tau \vee r_{1}\right)}^{2^{n}\left(\tau \wedge r_{2}\right)-1} \int_{t_{i}^{n}}^{t_{i+1}^{n}}\left(e_{j}\left(X_{T-s}\left(\omega^{\prime}\right)\right)-e_{j}\left(X_{T-t_{i}^{n}}\left(\omega^{\prime}\right)\right)\right)^{2} d s \\
& =\sum_{j=1}^{d} P\left(\Lambda_{j}\right) \int_{0}^{\tau^{2}\left(\tau \wedge r_{2}\right)-1} \sum_{i=2^{n}\left(\tau \vee r_{1}\right)} 1_{] t_{i}^{n}, t_{i+1}^{n}\right]}(s)\left(e_{j}\left(X_{T-s}\left(\omega^{\prime}\right)\right)-e_{j}\left(X_{T-t_{i}^{n}}\left(\omega^{\prime}\right)\right)\right)^{2} d s .
\end{aligned}
$$

Using the continuity of the process $s \rightarrow e_{j}\left(X_{T-s}\left(\omega^{\prime}\right)\right)$ for $m$-almost all $\omega^{\prime}$, we apply the dominated convergence theorem with respect to $d s \otimes P^{m}$ which yields

$$
\lim _{n \rightarrow+\infty} E^{m} E\left(d^{n}\right)^{2}=0 .
$$

To pass to the general case with $w$ arbitrary in $\left(\mathcal{P}\left(L^{2}\left(R^{N}\right)\right)\right)^{d}$, consider a sequence $\left(w^{n}\right)_{n>0}$ of combinations of elementary processes which converges to $w$ in $\left(\mathcal{P}\left(L^{2}\right)\right)^{\bar{d}}$. For all $n$, we set

$$
u_{t}^{n}=\sum_{j=1}^{d} \int_{0}^{t} P_{t-s} w_{j, s}^{n} d B_{s}^{j} .
$$

Then we deduce

$$
\begin{aligned}
& E^{m} E\left(\int_{0}^{t}\left(w_{j}-w_{j}^{n}\right)\left(\omega, s, X_{T-s}\left(\omega^{\prime}\right)\right) d B_{s}^{j}(\omega)\right)^{2} \\
= & E^{m} E \int_{0}^{t}\left(w_{j}-w_{j}^{n}\right)^{2}\left(\omega, s, X_{T-s}\left(\omega^{\prime}\right)\right) d s \\
= & E \int_{0}^{t} E^{m}\left(w_{j}-w_{j}^{n}\right)^{2}\left(\omega, s, X_{T-s}\left(\omega^{\prime}\right)\right) d s \\
= & E \int_{0}^{t}\left\|w_{j}-w_{j}^{n}\right\|^{2} d s,
\end{aligned}
$$

and

$$
\begin{aligned}
& E E^{m}\left(\sum_{i=1}^{N} \int_{T-t}^{T}\left(\partial_{i} u-\partial_{i} u^{n}\right)\left(\omega, T-s, X_{s}\left(\omega^{\prime}\right)\right) d M_{s}^{i}\left(\omega^{\prime}\right)\right)^{2} \\
= & E E^{m} \sum_{i, j=1}^{N} \int_{T-t}^{T} \partial_{i}\left(u-u^{n}\right) \partial_{j}\left(u-u^{n}\right)\left(\omega, T-s, X_{s}\left(\omega^{\prime}\right)\right) d\left\langle M^{i}, M^{j}\right\rangle_{s}\left(\omega^{\prime}\right) \\
= & E \int_{T-t}^{T} \mathcal{E}\left(u_{T-s}-u_{T-s}^{n}\right) d s .
\end{aligned}
$$

Then, we use the relation ( $i i)$ of Proposition 6 to conclude, because it is also clear that $u^{n}\left(\omega, t, X_{T-t}\left(\omega^{\prime}\right)\right)$ converges to $u\left(\omega, t, X_{T-t}\left(\omega^{\prime}\right)\right)$ in $L^{2}\left(R^{N}\right)$. 
Now we are going to interpret probabilistically the solutions of the nonlinear equation. We assume that the "concrete" functions from the first example in the introduction satisfy all the conditions mentioned there and that $2 \alpha+\beta^{2}<2$. Then the conditions to apply Theorem 8 are satisfied and one deduces the existence and uniqueness of the solution of the equation (1).

Corollary 12 Let $u$ be a solution of the equation (1). Then this solution admits a double stochastic representation as follows,

$$
\begin{gathered}
u\left(\omega, t, X_{T-t}\left(\omega^{\prime}\right)\right)=u_{0}\left(\omega, X_{T}\left(\omega^{\prime}\right)\right) \\
-\int_{0}^{t} \widetilde{f}\left(s, \omega, X_{T-s}\left(\omega^{\prime}\right), u\left(\omega, s, X_{T-s}\left(\omega^{\prime}\right)\right), \frac{1}{\sqrt{2}} \nabla u \sigma\left(\omega, s, X_{T-s}\left(\omega^{\prime}\right)\right)\right) d s \\
-\frac{1}{2} \sum_{i=1}^{N}\left[\int_{T-t}^{T} \widetilde{g}_{i}\left(T-s, \omega, X_{s}\left(\omega^{\prime}\right), u\left(\omega, T-s, X_{s}\left(\omega^{\prime}\right)\right), \frac{1}{\sqrt{2}} \nabla u \sigma\left(\omega, T-s, X_{s}\left(\omega^{\prime}\right)\right)\right) d M_{s}^{i}\left(\omega^{\prime}\right)\right. \\
\left.+\int_{0}^{t} \widetilde{g}_{i}\left(s, \omega, X_{T-s}\left(\omega^{\prime}\right), u\left(\omega, s, X_{T-s}\left(\omega^{\prime}\right)\right), \frac{1}{\sqrt{2}} \nabla u \sigma\left(\omega, s, X_{T-s}\left(\omega^{\prime}\right)\right)\right) d \overleftarrow{M}^{i}\left(\omega^{\prime}\right)\right] \\
-\sum_{i} \int_{T-t}^{T} \partial_{i} u\left(\omega, T-s, X_{s}\left(\omega^{\prime}\right)\right) d M_{s}^{i}\left(\omega^{\prime}\right) \\
+\sum_{j=1}^{d} \int_{0}^{t} \widetilde{h}_{j}\left(s, \omega, X_{T-s}\left(\omega^{\prime}\right), u\left(\omega, s, X_{T-s}\left(\omega^{\prime}\right)\right), \frac{1}{\sqrt{2}} \nabla u \sigma\left(\omega, s, X_{T-s}\left(\omega^{\prime}\right)\right)\right) d B_{s}^{j}(\omega) .
\end{gathered}
$$

Proof. Taking into account the formula of the mild equation (Definition 1) and the representation obtained in the preceding proposition, it is enough to prove a double stochastic representation for a process of the type

$$
v_{t}=P_{t} u_{0}-\int_{0}^{t} P_{t-s} w_{s} d s-\int_{0}^{t} P_{t-s} \sum_{i j} \partial_{i} a^{i j} w_{j, s} d s
$$

where $u_{0} \in L^{2}, w, w_{j} \in \mathcal{P}\left(L^{2}\right)$. But this process satisfies, on almost each trajectory, the following deterministic equation

$$
\left(\partial_{t}-L\right) v(\omega, t)+w(\omega, t)-\sum_{i j} \partial_{i} a^{i j} w_{j}(\omega, t)=0, v(\omega, 0)=u_{0}(\omega) .
$$

Its representation then follows from [18], namely one has

$$
\begin{gathered}
v\left(\omega, t, X_{T-t}\left(\omega^{\prime}\right)\right)=u_{0}\left(\omega, X_{T}\left(\omega^{\prime}\right)\right) \\
-\int_{0}^{t} w\left(\omega, s, X_{T-s}\left(\omega^{\prime}\right)\right) d s
\end{gathered}
$$




$$
\begin{gathered}
-\frac{1}{2} \sum_{i=1}^{N}\left[\int_{T-t}^{T} w_{i}\left(\omega, T-s, X_{s}\left(\omega^{\prime}\right)\right) d M_{s}^{i}\left(\omega^{\prime}\right)\right. \\
\left.\quad+\int_{0}^{t} w_{i}\left(\omega, s, X_{T-s}\left(\omega^{\prime}\right)\right) d \overleftarrow{M}^{i}\left(\omega^{\prime}\right)\right] \\
-\sum_{i} \int_{T-t}^{T} \partial_{i} v\left(\omega, T-s, X_{s}\left(\omega^{\prime}\right)\right) d M_{s}^{i}\left(\omega^{\prime}\right) .
\end{gathered}
$$

Acknowledgment The authors thank their friend Vlad Bally for introducing them to the area of s.p.d.e.'s.

\section{References}

[1] Bally V., Matoussi A. "Weak solutions for SPDEs and backward doubly stochastic differential equations", Journal of Theoretical Probability, Vol. 14, No 1, 2001.

[2] Bally V., Pardoux E., Stoica L. "Backward stochastic differential equations associated to a symmetric Markov process", to appear in Potential Analysis.

[3] Bouleau N., Hirsch F., "Dirichlet forms and analysis on Wiener space", Kluwer, 1993.

[4] Cardon-Weber C., "Cahn-Hilliard stochastic equation: existence of the solution and of its density", Bernoulli 7 (5), p777-816 (2001).

[5] Chojnowska-Michalik A., "Stochastic diffrential equations in Hilbert spaces", Probability theory, Banach center publications Vol. 5, 1979.

[6] Da Prato G., "Stochastic evolution equations by semigroups methods", Centre de Recerca Matematica, Quaderns, num. 11/gener 1998.

[7] Da Prato G., Zabczyk J. "Stochastic Equations in Infinite Dimensions", Encyclopedia of Mathematics and its Applications, Cambridge University Press, 1992.

[8] Davies, E.B. "One parameter semigroups", Academic Press, 1980.

[9] Fukushima M., Oshima Y., Takeda M. "Dirichlet Forms and Symmetric Markov Processes", de Gruyter studies in Math., 1994.

[10] Gyöngy I., Rovira C. "On $L^{p}$-solutions of semilinear stochastic partial differential equations", Stochastic Processes and their Applications 90 (2000), 83-108. 
[11] Krylov N.V. "An analytic approach to SPDEs. Stochastic partial differential Equations: six perspectives", AMS Mathematical Surveys and Monographs, 64, (1999) 185-242.

[12] Lions J.L. "Équations Différentielles opérationnelles", Springer -Verlag, Berlin -Göttingen -Heidelberg, 1961.

[13] Mikulevicius R., Rozovskii B.L. "A note on Krylov's Lp-theory for systems of SPDEs" Electronic Journal of Probability, 6, (2002) paper no.12, pp. 1-35.

[14] Pardoux E., "Stochastic PDE's and filtering of diffusion processes", Stochastics 3, 127-167 (1979).

[15] Pardoux E., Peng S. "Backward doubly stochastic differential equations and systems of quasilinear SPDEs", Prob. Th. Rel. Fields, 98, 209-227 (1994).

[16] Rozovskii B.L., "Stochastic Evolution Systems", Kluver, DordrechtBoston- London, 1990.

[17] Saussereau B. "Sur un Classe d'Equations aux Dérivées Partielles", thèse de Doctorat de l'Université du Maine, 2001.

[18] Stoica L., "A probabilistic representation of the divergence and $B S D E$ 's", Stochastic Processes and their Applications 103, (2003) pp.31-55.

[19] Walsh J.B. "An introduction to stochastic partial differential equations" in vol. Ecole d'Eté de Probabilités de Saint Flour, XIV, ed. P.L.Hennequin, L.N.M. no.1180, 265-439, Springer, 1984. 